\title{
Extrinsic Methods for Coding and Dictionary Learning on Grassmann Manifolds
}

\author{
Mehrtash Harandi, Richard Hartley, Chunhua Shen, Brian Lovell, Conrad Sanderson
}

- Published as:

\author{
M. Harandi, R. Hartley, C. Shen, B. Lovell, C. Sanderson. \\ Extrinsic Methods for Coding and Dictionary Learning on Grassmann Manifolds. \\ International Journal of Computer Vision, (in press). \\ http:/ / dx.doi.org/10.1007/s11263-015-0833-x
}

\begin{abstract}
Sparsity-based representations have recently led to notable results in various visual recognition tasks. In a separate line of research, Riemannian manifolds have been shown useful for dealing with features and models that do not lie in Euclidean spaces. With the aim of building a bridge between the two realms, we address the problem of sparse coding and dictionary learning in Grassmann manifolds, i.e., the space of linear subspaces. To this end, we propose to embed Grassmann manifolds into the space of symmetric matrices by an isometric mapping. This in turn enables us to extend two sparse coding schemes to Grassmann manifolds. Furthermore, we propose an algorithm for learning a Grassmann dictionary, atom by atom. Lastly, to handle non-linearity in data, we extend the proposed Grassmann sparse coding and dictionary learning algorithms through embedding into higher dimensional Hilbert spaces.

Experiments on several classification tasks (gender recognition, gesture classification, scene analysis, face recognition, action recognition and dynamic texture classification) show that the proposed approaches achieve considerable improvements in discrimination accuracy, in comparison to state-of-the-art methods such as kernelized Affine Hull Method and graph-embedding Grassmann discriminant analysis.
\end{abstract}

Keywords: Riemannian geometry, Grassmann manifolds, sparse coding, dictionary learning

\section{Affiliations}

Mehrtash Harandi and Rirchard Hartley

NICTA, Australia

and

College of Engineering and Computer Science, Australian National University

Chunhua Shen

School of Computer Science, University of Adelaide, Australia

Brian Lovell

University of Queensland, Brisbane, Australia

Conrad Sanderson

NICTA, Australia

and

University of Queensland, Brisbane, Australia 


\section{Introduction}

In the past decade, sparsity has become a popular term in neuroscience, information theory, signal processing and related areas $[51,7,17,70,19]$. Through sparse representation and compressive sensing it is possible to represent natural signals like images using only a few non-zero coefficients of a suitable basis. In computer vision, sparse and overcomplete image representations were first introduced for modeling the spatial receptive fields of simple cells in the human visual system by [51]. The linear decomposition of a signal using a few atoms of a dictionary has been shown to deliver notable results for various visual inference tasks, such as face recognition [70, 69], image classification [72, 47], subspace clustering [20], image restoration [48], and motion segmentation [53] to name a few. While significant steps have been taken to develop the theory of the sparse coding and dictionary learning in Euclidean spaces, similar problems on non-Euclidean geometry have received comparatively little attention [76, 32, 31, 29, 36, 10].

This paper introduces techniques to sparsely represent $p$-dimensional linear subspaces in $\mathbf{R}^{d}$ using a combination of linear subspaces. Linear subspaces can be considered as the core of many inference algorithms in computer vision and machine learning. For example, the set of all reflectance functions produced by Lambertian objects lies in a linear subspace $[5,52]$. Several state-of-the-art methods for matching videos or image sets model given data by subspaces $[30,33,63,65,56,13]$. Auto regressive and moving average models, which are typically employed to model dynamics in spatio-temporal processing, can also be expressed by linear subspaces [63]. More applications of linear subspaces in computer vision include, chromatic noise filtering [61], subspace clustering [20], motion segmentation [53], domain adaptation [25, 26], and object tracking [59].

Despite their wide applications and appealing properties, subspaces lie on a special type of Riemannian manifold, namely the Grassmann manifold, which makes their analysis very challenging. This paper tackles and provides efficient solutions to the following two fundamental problems on Grassmann manifolds (see Fig. 1 for a conceptual illustration):

1. Coding. Given a subspace $\mathcal{X}$ and a set $\mathbb{D}=\left\{\mathcal{D}_{i}\right\}_{i=1}^{N}$ with $N$ elements (also known as atoms), where $\mathcal{X}$ and $\mathcal{D}_{i}$ are linear subspaces, how can $\mathcal{X}$ be approximated by a combination of atoms in $\mathbb{D}$ ?

2. Dictionary learning. Given a set of subspaces $\left\{\mathcal{X}_{i}\right\}_{i=1}^{m}$, how can a smaller set of subspaces $\mathbb{D}=\left\{\mathcal{D}_{i}\right\}_{i=1}^{N}$ be learned to represent $\left\{\mathcal{X}_{i}\right\}_{i=1}^{m}$ accurately?

Our main motivation here is to develop new methods for analyzing video data and image sets. This is inspired by the success of sparse signal modeling and related topics that suggest natural signals like images (and hence video and image sets as our concern here) can be efficiently approximated by superposition of atoms of a dictionary. We generalize the traditional notion of coding, which operates on vectors, to coding on subspaces. Coding with the dictionary of subspaces can then be seamlessly used for categorizing video data.

Considering the problem of coding and dictionary learning on Grassmann manifolds, previous studies (e.g., $[36,9,10])$ opt for an intrinsic and general framework for sparse coding on Riemannian manifolds. This intrinsic formulation exploits the tangent bundle of the manifold for sparse coding. Due to the computational complexity of the logarithm map on Grassmann manifolds, performing intrinsic sparse coding might be computationally demanding for the problems that we are interested in (e.g., video analysis). Moreover, learning a dictionary based on the intrinsic formulation as proposed by [36] requires computing the gradient of a cost function that includes terms based on logarithm map. As will be shown later, the involvement of logarithm map (which does not have an analytic formulation on Grassmann manifolds) deprives us from having a closed-form solution for learning a Grassmann dictionary intrinsically. 


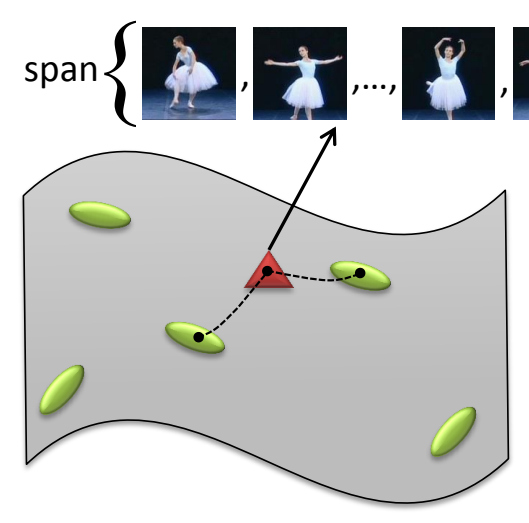

(a)

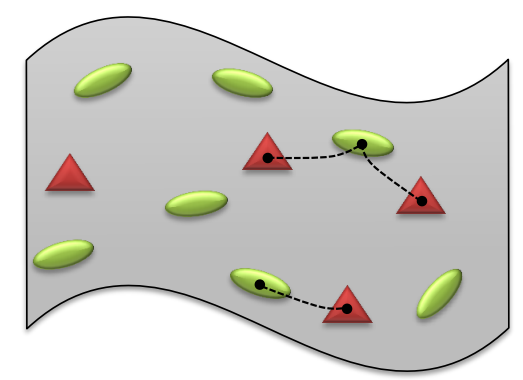

(b)

Figure 1: A conceptual diagram of the problems addressed in this work. A video or an image set can be modeled by a linear subspace, which can be represented as a point on a Grassmann manifold. (a) Sparse coding on a Grassmann manifold. Given a dictionary (green ellipses) and a query signal (red triangle) on the Grassmann manifold, we are interested in estimating the query signal by a sparse combination of atoms while taking into account the geometry of the manifold (e.g., curvature). (b) Dictionary learning on a Grassmann manifold. Given a set of observations (green ellipses) on a Grassmann manifold, we are interested in determining a dictionary (red triangles) to describe the observations sparsely, while taking into account the geometry. This figure is best seen in color.

\subsection{Contributions}

In light of the above discussion, in this paper we introduce an extrinsic methods for coding and dictionary learning on Grassmann manifolds. To this end, we propose to embed Grassmann manifolds into the space of symmetric matrices by a diffeomorphism that preserves several properties of the Grassmannian structure. We show how coding can be accomplished in the induced space and devise an algorithm for updating a Grassmann dictionary atom by atom. Furthermore, in order to accommodate non-linearity in data, we propose kernelized versions of our coding and dictionary learning algorithms. Our contributions are therefore three-fold:

1. We propose to perform coding and dictionary learning for data points on Grassmann manifolds by embedding the manifolds into the space of symmetric matrices.

2. We derive kernelized versions of the proposed coding and dictionary learning algorithms (i.e., embedded into Hilbert spaces), which can address non-linearity in data.

3. We apply the proposed Grassmann dictionary learning methods to several computer vision tasks where the data are videos or image sets. Our proposed algorithms outperform state-of-the-art methods on a wide range of classification tasks, including gender recognition from gait, scene analysis, face recognition from image sets, action recognition and dynamic texture classification.

\section{Background Theory}

This section overviews Grassmann geometry and provides the groundwork for techniques described in following sections. Since the term "manifold" itself is often used in computer vision in a somewhat loose sense, we emphasize that the word is used in this paper in its strict mathematical sense.

Throughout the paper, bold capital letters denote matrices (e.g. $\boldsymbol{X}$ ) and bold lower-case letters denote column vectors $(e . g . \boldsymbol{x})$. The notations $[\cdot]_{i}$ and $[\cdot]_{i, j}$ are used to demonstrate elements in position $i$ and $(i, j)$ in a vector and matrix, respectively. $\mathbf{1}_{d} \in \mathbf{R}^{d}$ and $\mathbf{0}_{d} \in \mathbf{R}^{d}$ are vectors of ones and zeros. $\mathbf{I}_{d}$ is the $d \times d$ identity matrix. $\|\boldsymbol{x}\|_{1}=\sum_{i}\left|[x]_{i}\right|$ and $\|\boldsymbol{x}\|=\sqrt{\boldsymbol{x}^{T} \boldsymbol{x}}$ denote the $\ell_{1}$ and $\ell_{2}$ norms, respectively, with $T$ indicating transposition. $\|\boldsymbol{X}\|_{F}=\sqrt{\operatorname{Tr}\left(\boldsymbol{X}^{T} \boldsymbol{X}\right)}$ designates the Frobenius norm, with $\operatorname{Tr}(\cdot)$ computing the matrix trace. 


\subsection{Grassmann Manifolds and their Riemannian Structure}

For $0<p \leq d$, the space of $d \times p$ matrices with orthonormal columns is not a Euclidean space but a Riemannian manifold, the Stiefel manifold $\operatorname{St}(p, d)$. That is,

$$
\operatorname{St}(p, d) \triangleq\left\{\boldsymbol{X} \in \mathbf{R}^{d \times p}: \boldsymbol{X}^{T} \boldsymbol{X}=\mathbf{I}_{p}\right\} .
$$

By grouping together all points on $\operatorname{St}(p, d)$ that span the same subspace we obtain the Grassmann manifold $\mathcal{G}(p, d)$. More formally, the Stiefel manifold $\operatorname{St}(p, d)$ admits a right action by the orthogonal group $O(p)$ (consisting of $p \times p$ orthogonal matrices); for $\boldsymbol{X} \in \operatorname{St}(p, d)$ and $\boldsymbol{U} \in O(p)$, the matrix $\boldsymbol{X} \boldsymbol{U}$ is also an element of $\operatorname{St}(p, d)$. Furthermore the columns of $\boldsymbol{X}$ and $\boldsymbol{X} \boldsymbol{U}$ span the same subspace of $\mathbf{R}^{d}$, and are to be thought of representatives of the same element of the Grassmann manifold, $\mathcal{G}(p, d)$. Thus, the orbits of this group action form the elements of the Grassman manifold. The resulting set of orbits is a manifold according to the quotient manifold theorem (see Theorem 21.10 in [42]). The details of this construction are not critical to an understanding of the rest of this paper.

An element $\mathcal{X}$ of $\mathcal{G}(p, d)$ can be specified by a basis, i.e., a set of $p$ vectors $\boldsymbol{x}_{1}, \cdots, \boldsymbol{x}_{p}$ such that $\mathcal{X}$ is the set of all their linear combinations. When the $\boldsymbol{x}$ vectors are ordered as the columns of a $d \times p$ matrix $\boldsymbol{X}$, then $\boldsymbol{X}$ is said to span $\mathcal{X}$ and we write $\mathcal{X}=\operatorname{span}(\boldsymbol{X})$. In what follows, we refer to a subspace $\mathcal{X}$ and hence a point on $\mathcal{G}(p, d)$ by its $d \times p$ basis matrix $\boldsymbol{X}$. The choice of the basis is not unique but it has no effect in what we develop later.

A Riemannian metric on a manifold is defined formally as a smooth inner product on the tangent bundle. (See [1] for the form of Riemannian metric on $\mathcal{G}(p, d)$ ). However, we shall be concerned only with geodesic distances on the Grassmann manifold, which allows us to avoid many technical points and give a straight-forward definition.

On a Riemannian manifold, points are connected via smooth curves. The geodesic distance between two points is defined as the length of shortest curve in the manifold (called a geodesic) connecting them. The Stiefel manifold $\operatorname{St}(p, d)$ is embedded in the set of $d \times p$ matrices, which may be seen as a Euclidean space $\mathbf{R}^{d \times p}$ with distances defined by the Frobenius norm. Consequently the length of a smooth curve (or path) in $\operatorname{St}(p, d)$ is defined as its length as a curve in $\mathbf{R}^{d \times p}$. Now, given two points $\mathcal{X}$ and $\mathcal{Y}$ in $\mathcal{G}(p, d)$, the distance $d_{\text {geod }}(\mathcal{X}, \mathcal{Y})$ is defined as the length of the shortest path in $\operatorname{St}(p, d)$ between any two points $\boldsymbol{X}$ and $\boldsymbol{Y}$ in $\operatorname{St}(p, d)$ that are members of the equivalence clases $\mathcal{X}$ and $\mathcal{Y}$.

The geodesic distance has an interpretation as the magnitude of the smallest rotation that takes one subspace to the other. If $\Theta=\left[\theta_{1}, \theta_{2}, \ldots, \theta_{p}\right]$ is the sequence of principal angles between two subspaces $\mathcal{X}_{1} \in \mathcal{G}(p, d)$ and $\mathcal{X}_{2} \in \mathcal{G}(p, d)$, then $d_{\text {geod }}\left(\mathcal{X}_{1}, \mathcal{X}_{2}\right)=\|\Theta\|_{2}$.

Definition 2.1 (Principal Angles). Let $\boldsymbol{X}_{1}$ and $\boldsymbol{X}_{2}$ be two matrices of size $d \times p$ with orthonormal columns. The principal angles $0 \leq \theta_{1} \leq \theta_{2} \leq \cdots \leq \theta_{p} \leq \pi / 2$ between two subspaces $\operatorname{Span}\left(\boldsymbol{X}_{1}\right)$ and $\operatorname{Span}\left(\boldsymbol{X}_{2}\right)$, are defined recursively by

$$
\begin{array}{cc} 
& \cos \left(\theta_{i}\right)=\max _{\boldsymbol{u}_{i} \in \operatorname{Span}\left(\boldsymbol{X}_{1}\right)} \max _{\boldsymbol{v}_{i} \in \operatorname{Span}\left(\boldsymbol{X}_{2}\right)} \boldsymbol{u}_{i}^{T} \boldsymbol{v}_{i} \\
\left\|\boldsymbol{u}_{i}\right\|_{2}=\left\|\boldsymbol{v}_{i}\right\|_{2}=1 \\
\text { s.t.: } \\
\boldsymbol{u}_{i}^{T} \boldsymbol{u}_{j}=0 ; j=1,2, \cdots, i-1 \\
\boldsymbol{v}_{i}^{T} \boldsymbol{v}_{j}=0 ; j=1,2, \cdots, i-1
\end{array}
$$

In other words, the first principal angle $\theta_{1}$ is the smallest angle between all pairs of unit vectors in the first and the second subspaces. The rest of the principal angles are defined similarly.

Two operators, namely the logarithm map $\log _{x}(\cdot): \mathcal{M} \rightarrow T_{\boldsymbol{x}}(\mathcal{M})$ and its inverse, the exponential map $\exp _{\boldsymbol{x}}(\cdot): T_{\boldsymbol{x}}(\mathcal{M}) \rightarrow \mathcal{M}$ are defined over Riemannian manifolds to switch between the manifold and the tangent space at $\boldsymbol{x}$. A key point here is the fact that both the logarithm map and its inverse do not have closed-form solutions for Grassmann manifolds. Efficient numerical approaches for computing both maps were proposed by $[21,6]$. In this paper, however, the exponential and logarithm maps will only be used when describing previous work of other authors. 


\section{Problem Statement}

In vector spaces, by coding we mean the general notion of representing a vector $\boldsymbol{x}$ (the query) as some combination of other vectors $\boldsymbol{d}_{i}$ belonging to a dictionary. Typically, $\boldsymbol{x}$ is expressed as a linear combination $\boldsymbol{x}=\sum_{j=1}^{N}[\boldsymbol{y}]_{j} \boldsymbol{d}_{j}$, or else as an affine combination in which the coefficients $[\boldsymbol{y}]_{j}$ satisfy the additional constraint $\sum_{j=1}^{N}[\boldsymbol{y}]_{j}=1$. (This constraint may also be written as $\mathbf{1}^{T} \boldsymbol{y}=1$.)

In sparse coding one seeks to express the query in terms of a small number of dictionary elements. Given a query $\boldsymbol{x} \in \mathbf{R}^{d}$ and a dictionary $\mathbb{D}$ of size $N$, i.e., $\mathbb{D}_{d \times N}=\left\{\boldsymbol{d}_{1}, \boldsymbol{d}_{2}, \cdots, \boldsymbol{d}_{N}\right\}$ with atoms $\boldsymbol{d}_{i} \in \mathbf{R}^{d}$, the problem of coding $\boldsymbol{x}$ can be formulated as solving the minimization problem:

$$
l_{E}(\boldsymbol{x}, \mathbb{D}) \triangleq \min _{\boldsymbol{y}}\left\|\boldsymbol{x}-\sum_{j=1}^{N}[\boldsymbol{y}]_{j} \boldsymbol{d}_{j}\right\|_{2}^{2}+\lambda f(\boldsymbol{y}) .
$$

The domain of $\boldsymbol{y}$ may be the whole of $\mathbf{R}^{N}$, so that the sum runs over all linear combinations of dictionary elements (or atoms), or alternatively, the extra constraint $\mathbf{1}^{T} \boldsymbol{y}$ may be specified, to restrict to affine combinations.

The idea here is to (approximately) reconstruct the query $\boldsymbol{x}$ by a combination of dictionary atoms while forcing the coefficients of combination, i.e., $\boldsymbol{y}$, to have some structure. The quantity $l_{E}(\boldsymbol{x}, \mathbb{D})$ can be thought of as a coding cost combining the squared residual coding error, reflected in the energy term $\|\cdot\|_{2}^{2}$ in (3), along with a penalty term $f(\boldsymbol{y})$, which encourages some structure such as sparsity. The function $f: \mathbf{R}^{N} \rightarrow \mathbf{R}$ could be the $\ell_{1}$ norm, as in the Lasso problem [62], or some form of locality as proposed by [74] and [67].

The problem of dictionary learning is to determine $\mathbb{D}$ given a finite set of observations $\left\{\boldsymbol{x}_{i}\right\}_{i=1}^{m}, \boldsymbol{x} \in \mathbf{R}^{d}$, by minimizing the total coding cost for all observations, namely

$$
h(\mathbb{D}) \triangleq \sum_{i=1}^{m} l_{E}\left(\boldsymbol{x}_{i}, \mathbb{D}\right)
$$

A "good" dictionary has a small residual coding error for all observations $\boldsymbol{x}_{i}$ while producing codes $\boldsymbol{y}_{i} \in \mathbf{R}^{N}$ with the desired structure. For example, in the case of sparse coding, the $\ell_{1}$ norm is usually taken as $f(\cdot)$ to obtain the most common form of dictionary learning in the literature. More specifically, the sparse dictionary learning problem may be written in full as that of jointly minimizing the total coding cost over all choices of coefficients and dictionary:

$$
\min _{\left\{\boldsymbol{y}_{i}\right\}_{i=1}^{m}, \mathbb{D}} \sum_{i=1}^{m}\left\|\boldsymbol{x}_{i}-\sum_{j=1}^{N}\left[\boldsymbol{y}_{i}\right]_{j} \boldsymbol{d}_{j}\right\|_{2}^{2}+\lambda \sum_{i=1}^{m}\left\|\boldsymbol{y}_{i}\right\|_{1} .
$$

A common approach to solving this is to alternate between the two sets of variables, $\mathbb{D}$ and $\left\{\boldsymbol{y}_{i}\right\}_{i=1}^{m}$, as proposed for example by [3] (see [19] for a detailed treatment). Minimizing (5) over sparse codes $\boldsymbol{y}_{i}$ while dictionary $\mathbb{D}$ is fixed is a convex problem. Similarly, minimizing the overall problem over $\mathbb{D}$ with fixed $\left\{\boldsymbol{y}_{i}\right\}_{i=1}^{m}$ is convex as well.

In generalizing the coding problem to a more general space $\mathcal{M}$, (e.g., Riemannian manifolds), one may write (3) as

$$
l_{\mathcal{M}}(\mathcal{X}, \mathbb{D}) \triangleq \min _{\boldsymbol{y}}\left(d_{\mathcal{M}}(\mathcal{X}, C(\boldsymbol{y}, \mathbb{D}))^{2}+\lambda f(\boldsymbol{y})\right) .
$$

Here $\mathcal{X}$ and $\mathbb{D}=\left\{\mathcal{D}_{j}\right\}_{j=1}^{N}$ are points in the space $\mathcal{M}$, while $d_{\mathcal{M}}(\cdot, \cdot)$ is some distance metric and $C$ : $\mathbf{R}^{N} \times \mathcal{M}^{N} \rightarrow \mathcal{M}$ is an encoding function, assigning an element of $\mathcal{M}$ to every choice of coefficients and dictionary. Note that (3) is a special case of this, in which $C(\boldsymbol{y}, \mathbb{D})$ represents linear or affine combination, and $d_{\mathcal{M}}(\cdot, \cdot)$ is the Euclidean distance metric. To define the coding, one need only specify the metric $d_{\mathcal{M}}(\cdot, \cdot)$ to be used and the encoding function $C(\cdot, \cdot)$. Although this formulation may apply to a wide range of spaces, here we shall be concerned chiefly with coding on Grassmann manifolds. 


\section{Related Work}

A seemingly straightforward method for coding and dictionary learning is through embedding manifolds into Euclidean spaces via a fixed tangent space. The embedding function in this case would be $\log _{\mathcal{P}}(\cdot)$, where $\mathcal{P}$ is some default base point. The natural choice for the base point on $\mathcal{G}(p, d)$ is

$$
\mathcal{P}=\operatorname{span}\left(\left[\begin{array}{c}
\mathbf{I}_{p \times p} \\
\mathbf{0}_{(d-p) \times p}
\end{array}\right]\right) .
$$

By mapping points in the manifold $\mathcal{M}$ to the tangent space, the problem at hand is transformed to its Euclidean counterpart. For example in the case of sparse coding, instead of (6), the encoding cost may be defined as follows:

$$
l_{\mathcal{M}}(\mathcal{X}, \mathbb{D}) \triangleq \min _{\boldsymbol{y}}\left\|\log _{\mathcal{P}}(\mathcal{X})-\sum_{j=1}^{N}[\boldsymbol{y}]_{j} \log _{\mathcal{P}}\left(\mathcal{D}_{j}\right)\right\|_{\mathcal{P}}^{2}+\lambda f(\boldsymbol{y})
$$

where the notation $\|\cdot\|_{\mathcal{P}}$ reminds us that the norm is in the tangent space at $\mathcal{X}$. We shall refer to this straightforward approach as Log-Euclidean sparse coding (the corresponding steps for Grassmann manifolds in Algorithm 1), following the terminology used in [4]. This idea has been deployed for action recognition on the manifold of Symmetric Positive Definite matrices by [76] and [29]. Since on a tangent space only distances to the base point are equal to true geodesic distances, the Log-Euclidean solution does not take into account the true structure of the underlying Riemannian manifold. Moreover, the solution is dependent upon the particular point $\mathcal{P}$ used as a base point.

A more elegant and intrinsic approach is to work in the tangent bundle of the manifold, varying the particular tangent space according to the point $\mathcal{X}$ being approximated. Such an idea has roots in the work of [23] which extends various methods of dimensionality reduction to Riemannian manifolds. As for sparse coding, [9], [10] and [36] show that by working in the tangent space at $\mathcal{X}$, i.e., $T_{\mathcal{X}}(\mathcal{M})$, the encoding cost in (6) can be written as

$$
l_{\mathcal{M}}(\mathcal{X}, \mathbb{D}) \triangleq \min _{\substack{\boldsymbol{y} \in \mathbf{R}^{N} \\ \mathbf{1}^{T} \boldsymbol{y}=1}}\left\|\sum_{j=1}^{N}[\boldsymbol{y}]_{j} \log _{\mathcal{X}}\left(\mathcal{D}_{j}\right)\right\|_{\mathcal{X}}^{2}+\lambda f(\boldsymbol{y})
$$

To see the relationship between (6) and (8), note that $\log _{\mathcal{X}}\left(\mathcal{D}_{j}\right)$ is unambiguously defined for most pairs $\left(\mathcal{X}, \mathcal{D}_{j}\right)$. If the encoding function $C(\cdot, \cdot)$ is defined by

$$
C(\boldsymbol{y}, \mathbb{D})=\exp _{\mathcal{X}}\left(\sum_{j=1}^{N}[\boldsymbol{y}]_{j} \log _{\mathcal{X}}\left(\mathcal{D}_{j}\right)\right)
$$

then

$$
\left\|\sum_{j=1}^{N}[\boldsymbol{y}]_{j} \log _{\mathcal{X}}\left(\mathcal{D}_{j}\right)\right\|_{\mathcal{X}}=\left\|\log _{\mathcal{X}} C(\boldsymbol{y}, \mathbb{D})\right\|_{\mathcal{X}}=d_{\text {geod }}(\mathcal{X}, C(\boldsymbol{y}, \mathbb{D})) .
$$

The extra affine constraint, i.e., $\mathbf{1}^{T} \boldsymbol{y}=1$ is necessary to avoid a trivial solution and has been used successfully in other applications such as dimensionality reduction [55], subspace clustering [20] and coding [73, 67] to name a few.

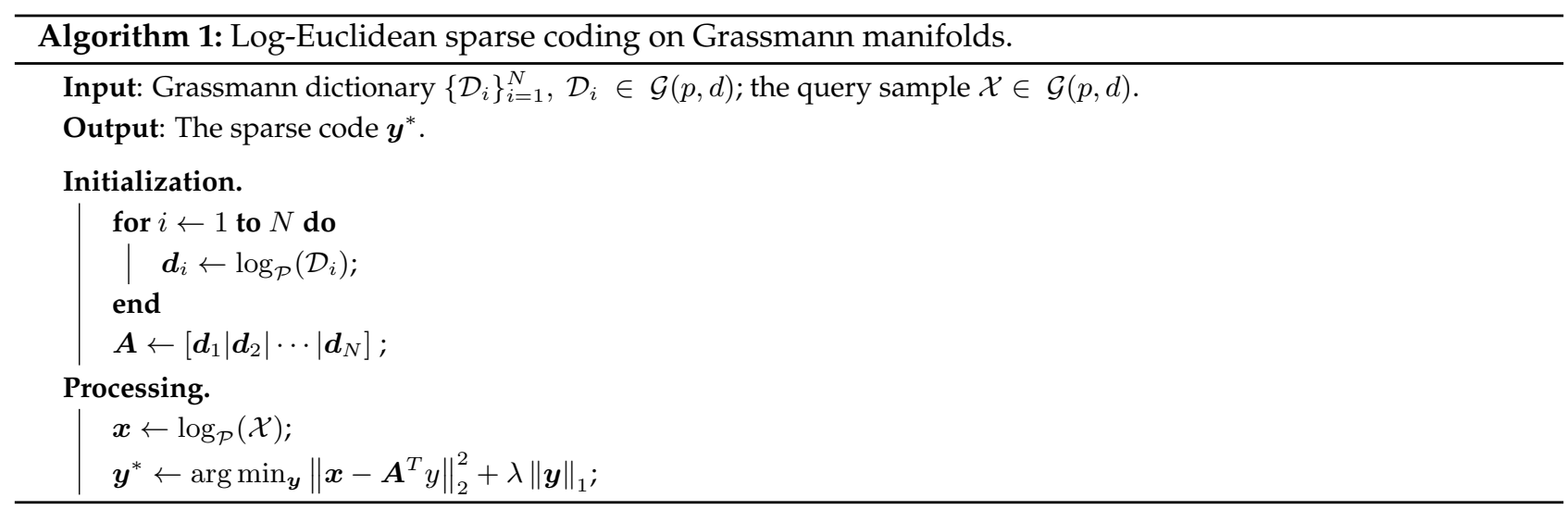


Turning our attention to the problem of dictionary learning, given a set of training data $\left\{\mathcal{X}_{i}\right\}_{i=1}^{m}, \mathcal{X}_{i} \in \mathcal{M}$, recasting the problem of (5) to the Riemannian manifold $\mathcal{M}$ by following [36] results in

$$
\begin{aligned}
& \min _{\left\{\boldsymbol{y}_{i}\right\}_{i=1}^{m}, \mathbb{D}} \sum_{i=1}^{m}\left\|\sum_{j=1}^{N}\left[\boldsymbol{y}_{i}\right]_{j} \log _{\mathcal{X}_{i}}\left(\mathcal{D}_{j}\right)\right\|^{2}+\lambda \sum_{i=1}^{m}\left\|\boldsymbol{y}_{i}\right\|_{1} \\
& \text { s.t. } \mathbf{1}^{T} \boldsymbol{y}_{i}=1, i=1,2, \cdots, m .
\end{aligned}
$$

Similar to the Euclidean case, the problem in (9) is solved by iterative optimization over $\left\{\boldsymbol{y}_{i}\right\}_{i=1}^{m}$ and $\mathbb{D}$. Computing the sparse codes $\left\{\boldsymbol{y}_{i}\right\}_{i=1}^{m}$ is done by solving (8). To update $\mathbb{D}$, [36] proposed a gradient descent approach along geodesics. That is, the update of $\mathcal{D}_{r}$ at time $t$ while $\left\{\boldsymbol{y}_{i}\right\}_{i=1}^{m}$ and $\mathcal{D}_{j}, j \neq r$ are kept fixed has the form

$$
\mathcal{D}_{r}^{(t)}=\exp _{\mathcal{D}_{r}^{(t-1)}}(-\eta \Delta) .
$$

In Eq. (10) $\eta$ is a step size and the tangent vector $\Delta: \mathbf{R} \rightarrow T_{\mathcal{D}_{r}}(\mathcal{M})$ represents the direction of maximum ascent. That is $\Delta=\operatorname{grad} \mathcal{J}\left(\mathcal{D}_{r}\right)^{1}$, where

$$
\mathcal{J}=\sum_{i=1}^{m}\left\|\sum_{j=1}^{N}\left[\boldsymbol{y}_{i}\right]_{j} \log _{\mathcal{X}_{i}}\left(\mathcal{D}_{j}\right)\right\|^{2}
$$

Here is where the difficulty arises. Since the logarithm map does not have a closed-form expression on Grassmann manifolds, an analytic expression for $\Delta$ in Eq. (10) cannot be sought for the case of interest in this work, i.e., Grassmann manifolds ${ }^{2}$. Having this in mind, we propose extrinsic approaches to coding and dictionary learning specialized for Grassmann manifolds. Our proposal is different from the intrinsic method in following points:

- As compared to the intrinsic approach, our extrinsic coding methods are noticeably faster. This is especially attractive for vision applications where the dimensionality of Grassmann manifolds is high.

- Similar to the intrinsic method, our proposed dictionary learning approach is an alternating method. However and in contrast to the intrinsic method, the updating rule for dictionary atoms admits an analytic form.

- Our proposed extrinsic methods can be kernelized. Such kernelization for the intrinsic method is not possible due to the fact that the logarithm map does not have a closed-form and analytic expression on Grassmann manifolds. Kernelized coding enables us to model non-linearity in data better (think of samples that do not lie on a subspace in low-dimensional space but could form one in a higher-possibly infinite- dimensional space). As shown in our experiments, kernelized coding can result in higher recognition accuracies as compared to linear coding.

\footnotetext{
${ }^{1}$ On an abstract Riemannian manifold $\mathcal{M}$, the gradient of a smooth real function $f$ at a point $x \in \mathcal{M}$, denoted by $\operatorname{grad} f(x)$, is the element of $T_{x}(\mathcal{M})$ satisfying $\langle\operatorname{grad} f(x), \zeta\rangle_{x}=D f_{x}[\zeta]$ for all $\zeta \in T_{x}(\mathcal{M})$. Here, $D f_{x}[\zeta]$ denotes the directional derivative of $f$ at $x$ in the direction of $\zeta$. The interested reader is referred to [2] for more details on how the gradient of a function on Grassmann manifolds can be computed.

${ }^{2}$ This is acknowledged by [36].
} 


\section{Coding on Grassmann Manifolds}

In this work, we propose to embed Grassmann manifolds into the space of symmetric matrices via the projection embedding [14]. The projection embedding has been previously used in subspace tracking [60], clustering [8], discriminant analysis [30,33] and classification purposes [65]. Let $\mathcal{P G}(p, d)$ be the set of $d \times d$ idempotent and symmetric matrices of rank $p$. The projection embedding $\Pi: \mathcal{G}(p, d) \rightarrow \mathcal{P} \mathcal{G}(p, d)$ is given by $\Pi(\mathcal{X})=\boldsymbol{X} \boldsymbol{X}^{T}$ where $\mathcal{X}=\operatorname{span}(\boldsymbol{X})$. The mapping $\Pi$ is a diffeomorphism [14], and $\mathcal{P G}(p, d)$ may be thought of as simply an alternative form of the Grassmann manifold. It is a smooth, compact submanifold of Sym(d) of dimension $d(d-p)[35]$.

From its embedding in $\operatorname{Sym}(d)$, the manifold $\mathcal{P G}(p, d)$ inherits a Riemannian metric (and hence a notion of path length), from the Frobenius norm in $\operatorname{Sym}(d)$. It is an important fact that the mapping $\Pi$ is also an isometry with respect to the Riemannian metric on $\mathcal{P G}(p, d)$ and the standard Riemannian metric, defined in Section 2 for $\mathcal{G}(p, d)$ [14]. Hence, $\Pi$ preserves length of curves [31]. The shortest path length between two points in $\mathcal{P} \mathcal{G}(p, d)$ defines a distance metric called the geodesic metric.

Working with $\mathcal{P G}(p, d)$ instead of $\mathcal{G}(p, d)$ has the advantage that each element of $\mathcal{P G}(p, d)$ is a single matrix, whereas elements of $\mathcal{G}(p, d)$ are equivalence classes of matrices. In other words, if $\boldsymbol{X}$ and $\boldsymbol{X}^{\star}=\boldsymbol{X} \boldsymbol{R}, \boldsymbol{R} \in \mathrm{O}(p)$ are two bases for $\mathcal{X}$, then $\Pi(\boldsymbol{X})=\Pi\left(\boldsymbol{X}^{\star}\right)$.

In future, we shall denote $\boldsymbol{X} \boldsymbol{X}^{T}$ by $\widehat{\boldsymbol{X}}$, the hat representing the action of the projection embedding. Furthermore, $\langle\cdot, \cdot\rangle$ represents the Frobenius inner product: thus $\langle\widehat{\boldsymbol{X}}, \widehat{\boldsymbol{Y}}\rangle=\operatorname{Tr}(\widehat{\boldsymbol{X}} \widehat{\boldsymbol{Y}})$. Note that in computing $\langle\widehat{\boldsymbol{X}}, \widehat{\boldsymbol{Y}}\rangle$ it is not necessary to compute $\widehat{\boldsymbol{X}}$ and $\widehat{\boldsymbol{Y}}$ explicitly (they may be large matrices). Instead, note that $\langle\widehat{\boldsymbol{X}}, \widehat{\boldsymbol{Y}}\rangle=\operatorname{Tr}(\widehat{\boldsymbol{X}} \widehat{\boldsymbol{Y}})=\operatorname{Tr}\left(\boldsymbol{X} \boldsymbol{X}^{T} \boldsymbol{Y} \boldsymbol{Y}^{T}\right)=\operatorname{Tr}\left(\boldsymbol{Y}^{T} \boldsymbol{X} \boldsymbol{X}^{T} \boldsymbol{Y}\right)=\left\|\boldsymbol{Y}^{T} \boldsymbol{X}\right\|_{F}^{2}$. This is advantageous, since $\boldsymbol{Y}^{T} \boldsymbol{X}$ may be a substantially smaller matrix.

Apart from the geodesic distance metric, an important metric used in this paper is the chordal metric:

$$
d_{\text {chord }}(\widehat{\boldsymbol{X}}, \widehat{\boldsymbol{Y}})=\|\Pi(\mathcal{X})-\Pi(\mathcal{Y})\|_{F}=\|\widehat{\boldsymbol{X}}-\widehat{\boldsymbol{Y}}\|_{F}
$$

This metric will be used in the context of (6) to recast the coding and consequently dictionary-learning problem in terms of chordal distance. Before presenting our proposed methods, we establish an interesting link between coding and the notion of weighted mean in a metric space.

\subsection{Weighted Karcher mean}

The underlying concept of coding using a dictionary is to represent in some way a point in a space of interest as a combination of other elements in that space. In the usual method of coding in $\mathbf{R}^{d}$ given by (3), each $\boldsymbol{x}$ is represented by a linear combination of dictionary elements $\boldsymbol{d}_{j}$, where the first term represents the coding error. For coding in a manifold, the problem to address is that linear combinations do not make sense. We wish to find some way in which an element $\mathcal{X}$ may be represented in terms of other dictionary elements $\mathcal{D}_{j}$ as suggested in (6). For a proposed method to generalize the $\mathbf{R}^{d}$ case, one may prefer a method that is a direct generalization of the Euclidean case in some way.

In $\mathbf{R}^{d}$, a different way to consider the expression $\sum_{j=1}^{N}[\boldsymbol{y}]_{j} \boldsymbol{d}_{j}$ in (3) is as a weighted mean of the points $\boldsymbol{d}_{j}$ This observation relies on the following fact, which is verified using a Lagrange multiplier method.

Lemma 5.1. Given coefficients $\boldsymbol{y}$ with $\sum_{i=1}^{N}[\boldsymbol{y}]_{i}=1$, and dictionary elements $\left\{\boldsymbol{d}_{1}, \ldots \boldsymbol{d}_{N}\right\}$ in $\mathbf{R}^{d}$, the point $\boldsymbol{x}^{*} \in \mathbf{R}^{d}$ that minimizes $\sum_{i=1}^{N}[\boldsymbol{y}]_{i}\left\|\boldsymbol{x}-\boldsymbol{d}_{i}\right\|_{F}^{2}$ is given by $\boldsymbol{x}^{*}=\sum_{i=1}^{N}[\boldsymbol{y}]_{i} \boldsymbol{d}_{i}$.

In other words, the affine combination of dictionary elements is equal to their weighted mean. Although linear combinations are not defined for points on manifolds or metric spaces, a weighted mean is.

Definition 5.1. Given points $\mathcal{D}_{i}$ on a Riemannian manifold $\mathcal{M}$, and weights $[\boldsymbol{y}]_{i}$, the point $\mathcal{X}^{*}$ that minimizes $\sum_{i=1}^{N}[\boldsymbol{y}]_{i} d_{g}\left(\mathcal{X}, \mathcal{D}_{i}\right)^{2}$, is called the weighted Karcher mean of the points $\mathcal{D}_{i}$ with weights $[\boldsymbol{y}]_{i}$. Here, $d_{g}(\cdot, \cdot)$ is the geodesic distance on $\mathcal{M}$. 
Generally, finding the Karcher mean [37] on a manifold involves an iterative procedure, which may converge to a local minimum, even on a simple manifold, such as $S O(3)[49,34]$. However, one may replace the geodesic metric with a different metric in order to simplify the calculation. To this end, we propose the chordal metric on a Grassman manifold, defined for matrices $\widehat{\boldsymbol{X}}$ and $\widehat{\boldsymbol{Y}}$ in $\mathcal{P} \mathcal{G}(p, n)$ by

$$
d_{\text {chord }}(\widehat{\boldsymbol{X}}, \widehat{\boldsymbol{Y}})=\|\widehat{\boldsymbol{X}}-\widehat{\boldsymbol{Y}}\|_{F} .
$$

The corresponding mean, as in definition 5.1 (but using the chordal metric) is called the weighted chordal mean of the points. In contrast to the Karcher mean, the weighted chordal mean on a Grassman manifold has a simple closed-form.

Theorem 5.1. The weighted chordal mean of a set of points $\widehat{\boldsymbol{D}}_{i} \in \mathcal{P} \mathcal{G}(p, d)$ with weights $[\boldsymbol{y}]_{i}$ is equal to $\operatorname{Proj}\left(\sum_{i=1}^{m}[\boldsymbol{y}]_{i} \widehat{\boldsymbol{D}}_{i}\right)$, where $\operatorname{Proj}(\cdot)$ represents the closest point on $\mathcal{P} \mathcal{G}(p, d)$.

The function $\operatorname{Proj}(\cdot)$ has a closed form solution in terms of the Singular Value Decomposition. For proofs of these results, see the proofs of Theorems A.1 and A.2 in the appendix.

The chordal metric on a Grassman manifold is not a geodesic metric (that is it is not equal to the length of a shortest geodesic under the Riemannian metric). However, it is closely related. In fact, one may easily show that for $\mathcal{G}(p, d) \ni \mathcal{X}=\operatorname{span}(\boldsymbol{X})$ and $\mathcal{G}(p, d) \ni \mathcal{Y}=\operatorname{span}(\boldsymbol{Y})$

$$
\frac{2}{\pi} d_{\text {geod }}(\mathcal{X}, \mathcal{Y}) \leq d_{\text {chord }}(\widehat{\boldsymbol{X}}, \widehat{\boldsymbol{Y}}) \leq d_{\text {geod }}(\mathcal{X}, \mathcal{Y}) .
$$

Furthermore, the path-metric [34] induced by $d_{\mathrm{chord}}(\cdot, \cdot)$ is equal to the geodesic distance.

\subsection{Sparse Coding}

Given a dictionary $\mathbb{D}$ with atoms $\widehat{\boldsymbol{D}}_{j} \in \mathcal{P} \mathcal{G}(p, d)$ and a query sample $\widehat{\boldsymbol{X}}$ the problem of sparse coding can be recast extrinsically as (see Fig. 2 for a conceptual illustration):

$$
l(\mathcal{X}, \mathbb{D}) \triangleq \min _{\boldsymbol{y}}\left\|\widehat{\boldsymbol{X}}-\sum_{j=1}^{N}[\boldsymbol{y}]_{j} \widehat{\boldsymbol{D}}_{j}\right\|_{F}^{2}+\lambda\|\boldsymbol{y}\|_{1} .
$$

The formulation here varies slightly from the general form given in (6), in that the point $\sum_{j=1}^{N}[\boldsymbol{y}]_{j} \widehat{\boldsymbol{D}}_{j}$ does not lie exactly on the manifold $\mathcal{P G}(p, d)$, since it is not idempotent nor its rank is necessarily $p$. We call this solution an extrinsic solution; the point coded by the dictionary is allowed to step out of the manifold. There is no reason to see this as a major flaw, as will be discussed later in Section 5.5.

Expanding the Frobenius norm term in (14) results in a convex function in $y$ :

$$
\left\|\widehat{\boldsymbol{X}}-\sum_{j=1}^{N}[\boldsymbol{y}]_{j} \widehat{\boldsymbol{D}}_{j}\right\|_{F}^{2}=\|\widehat{\boldsymbol{X}}\|_{F}^{2}+\left\|\sum_{j=1}^{N}[\boldsymbol{y}]_{j} \widehat{\boldsymbol{D}}_{j}\right\|_{F}^{2}-2\left\langle\sum_{j=1}^{N}[\boldsymbol{y}]_{j} \widehat{\boldsymbol{D}}_{j}, \widehat{\boldsymbol{X}}\right\rangle .
$$

The sparse codes can be obtained without explicit embedding of the manifold to $\mathcal{P G}(p, d)$ using $\Pi(\mathcal{X})$. This can be seen by defining $[\mathcal{K}(\boldsymbol{X}, \mathbb{D})]_{i}=\left\langle\widehat{\boldsymbol{X}}, \widehat{\boldsymbol{D}}_{i}\right\rangle$ as an $N$ dimensional vector storing the similarity between signal $\boldsymbol{X}$ and dictionary atoms in the induced space and $[\mathbb{K}(\mathbb{D})]_{i, j}=\left\langle\widehat{\boldsymbol{D}}_{i}, \widehat{\boldsymbol{D}}_{j}\right\rangle$ as an $N \times N$ symmetric matrix encoding the similarities between dictionary atoms (which can be computed offline). Then, the sparse coding in (14) can be written as:

$$
l(\mathcal{X}, \mathbb{D})=\min _{\boldsymbol{y}} \boldsymbol{y}^{T} \mathbb{K}(\mathbb{D}) \boldsymbol{y}-2 \boldsymbol{y}^{T} \mathcal{K}(\boldsymbol{X}, \mathbb{D})+\lambda\|\boldsymbol{y}\|_{1}
$$

The symmetric matrix $\mathbb{K}(\mathbb{D})$ is positive semidefinite since for all $\boldsymbol{v} \in \mathbf{R}^{N}$ :

$$
\begin{aligned}
\boldsymbol{v}^{T} \mathbb{K}(\mathbb{D}) \boldsymbol{v} & =\sum_{i=1}^{N} \sum_{j=1}^{N} v_{i} v_{j}\left\langle\widehat{\boldsymbol{D}}_{i}, \widehat{\boldsymbol{D}}_{j}\right\rangle=\left\langle\sum_{i=1}^{N} v_{i} \widehat{\boldsymbol{D}}_{i}, \sum_{j=1}^{N} v_{j} \widehat{\boldsymbol{D}}_{j}\right\rangle \\
& =\left\|\sum_{i=1}^{N} v_{i} \widehat{\boldsymbol{D}}_{i}\right\|_{F}^{2} \geq 0 .
\end{aligned}
$$

Therefore, the problem is convex and can be efficiently solved using common packages like CVX [28, 27] or SPAMS [46]. The problem in (15) can be transposed into a vectorized sparse coding problem. More specifically, let $\boldsymbol{U} \boldsymbol{\Sigma} \boldsymbol{U}^{T}$ be the SVD of $\mathbb{K}(\mathbb{D})$. Then (15) is equivalent to

$$
l(\mathcal{X}, \mathbb{D})=\min _{\boldsymbol{y}}\left\|\boldsymbol{x}^{*}-\boldsymbol{A} \boldsymbol{y}\right\|^{2}+\lambda\|\boldsymbol{y}\|_{1},
$$


where $\boldsymbol{A}=\boldsymbol{\Sigma}^{1 / 2} \boldsymbol{U}^{T}$ and $\boldsymbol{x}^{*}=\boldsymbol{\Sigma}^{-1 / 2} \boldsymbol{U}^{T} \mathcal{K}(\boldsymbol{X}, \mathbb{D})$. This can be easily verified by plugging $\boldsymbol{A}$ and $\boldsymbol{x}^{*}$ into (16). Algorithm 2 provides the pseudo-code for performing Grassmann Sparse Coding (gSC).

A special case is sparse coding on the Grassmann manifold $\mathcal{G}(1, d)$, which can be seen as a problem on $d-1$ dimensional unit sphere, albeit with a subtle difference. More specifically, unlike conventional sparse coding in vector spaces, $\boldsymbol{x} \sim-\boldsymbol{x}, \forall \boldsymbol{x} \in \mathcal{G}(1, d)$, which results in having antipodals points being equivalent. For this special case, the solution proposed in (14) can be understood as sparse coding in the higher dimensional quadratic space, i.e., $f: \mathbf{R}^{d} \rightarrow \mathbf{R}^{d^{2}}, f(\boldsymbol{x})=\left[x_{1}^{2}, x_{1} x_{2}, \cdots, x_{d}^{2}\right]^{T}$. We note that in the quadratic space, $\|f(\boldsymbol{x})\|=1$ and $f(\boldsymbol{x})=f(-\boldsymbol{x})$.

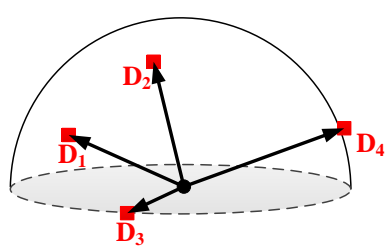

(a)

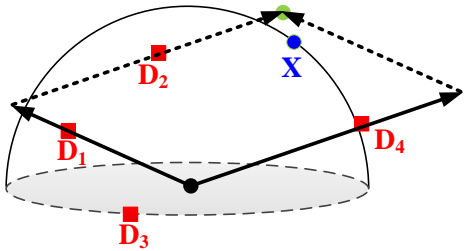

(b)

Figure 2: A conceptual diagram of the extrinsic sparse coding addressed in this work. The hemisphere is being used to represent $\mathcal{P G}(p, d)$. Each point on the surface of the hemisphere is intended to be a Grassmannian point represented by a symmetric, idempotent and rank $p$ matrix. (a) A Grassmann dictionary on $\mathcal{P G}(p, d)$ with four atoms (red squares). (b) Sparsely describing a query point shown by a blue circle using dictionary atoms. Here, the combination of atoms (green circle) could step out of $\mathcal{P G}(p, d)$. Having an overcomplete dictionary (enough atoms), it is possible to get arbitrarily close to the manifold. This is in spirit similar to sparse coding in vector spaces. More specifically, for a unit norm vector $\boldsymbol{x} \in \mathbf{R}^{d}$ and a dictionary $\mathbb{D}=\left\{\boldsymbol{d}_{i}\right\}$ with unit norm atoms, the result of sparse coding might be outside the unit norm sphere in $\mathbf{R}^{d}$.

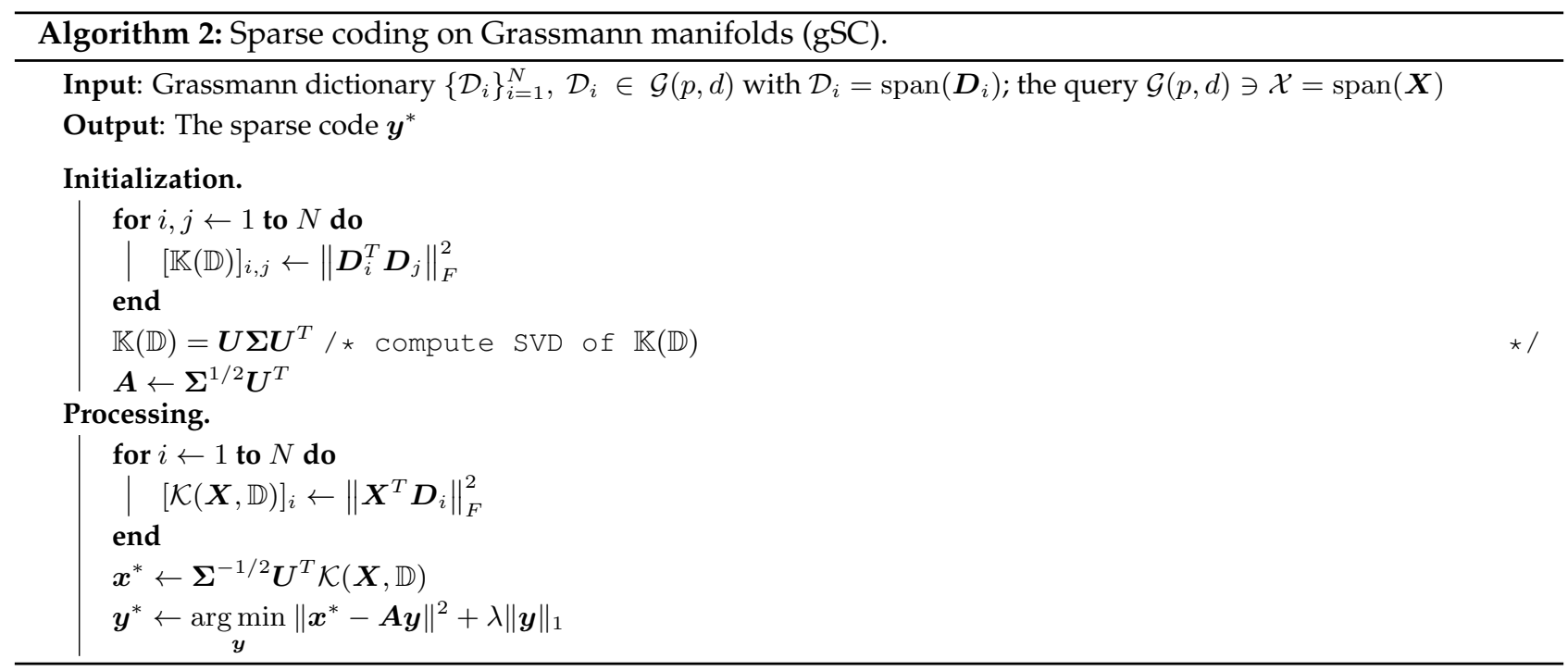




\subsection{Locality-Constrained Coding}

Several studies favor locality in coding process as locality could lead to sparsity but not necessarily vice versa $[55,74,67]$. In what follows, we describe a coding scheme based on neighborhood information. We show that the codes with local constraints can be obtained in closed-form which in turn avoids convex optimization problems as required for sparse coding. However, there is no free lunch here since the new algorithm requires a new parameter, namely the number of nearest neighbors, to generate the codes.

In vector spaces, a fast version of Locality-constrained Linear Coding (LLC) [67] is described by:

$$
\begin{aligned}
& \min _{\boldsymbol{y}}\|\boldsymbol{x}-\boldsymbol{B} \boldsymbol{y}\|^{2} \\
& \text { s.t. } \mathbf{1}^{\mathrm{T}} \boldsymbol{y}=1 .
\end{aligned}
$$

In (17), $\boldsymbol{x} \in \mathbf{R}^{d}$ is the query, $\boldsymbol{B} \in \mathbf{R}^{d \times N_{L C}}$ is a local basis obtained by simply stacking the $N_{L C}$ nearest neighbors of $\boldsymbol{x}$ from a global dictionary $\mathbb{D} \in \mathbf{R}^{d \times N}$ and $\boldsymbol{y}$ is the $N_{L C}$ dimensional LLC vector. Recasting the LLC problem depicted in (17) to Grassmann manifolds using the mapping $\Pi(\cdot)$, we obtain:

$$
\begin{array}{ll}
\min _{\boldsymbol{y}} & \left\|\widehat{\boldsymbol{X}}-\sum_{j=1}^{N_{L C}}[\boldsymbol{y}]_{j} \widehat{\boldsymbol{B}}_{j}\right\|_{F}^{2} \\
\text { s.t. } & \mathbf{1}^{\mathrm{T}} \boldsymbol{y}=1 .
\end{array}
$$

Observing the constraint $\mathbf{1}^{T} \boldsymbol{y}=1$, we may write

$$
\begin{aligned}
\left\|\widehat{\boldsymbol{X}}-\sum_{j=1}^{N_{L C}}[\boldsymbol{y}]_{j} \widehat{\boldsymbol{B}}_{j}\right\|_{F}^{2} & =\left\|\sum_{j=1}^{N_{L C}}[\boldsymbol{y}]_{j}\left(\widehat{\boldsymbol{X}}-\widehat{\boldsymbol{B}}_{j}\right)\right\|_{F}^{2} \\
& =\left\langle\sum_{i=1}^{N_{L C}}[\boldsymbol{y}]_{i}\left(\widehat{\boldsymbol{X}}-\widehat{\boldsymbol{B}}_{i}\right), \sum_{j=1}^{N_{L C}}[\boldsymbol{y}]_{j}\left(\widehat{\boldsymbol{X}}-\widehat{\boldsymbol{B}}_{j}\right)\right\rangle \\
& =\sum_{i, j=1}^{N_{L C}}[\boldsymbol{y}]_{i}[\boldsymbol{y}]_{j}\left\langle\widehat{\boldsymbol{X}}-\widehat{\boldsymbol{B}}_{i}, \widehat{\boldsymbol{X}}-\widehat{\boldsymbol{B}}_{j}\right\rangle \\
& =\boldsymbol{y}^{T} \mathbb{B} \boldsymbol{y}
\end{aligned}
$$

where the elements of matrix $\mathbb{B}$ are

$$
\begin{aligned}
{[\mathbb{B}]_{i, j} } & =\left\langle\widehat{\boldsymbol{X}}-\widehat{\boldsymbol{B}}_{i}, \widehat{\boldsymbol{X}}-\widehat{\boldsymbol{B}}_{j}\right\rangle \\
& =p-\left\|\boldsymbol{X}^{T} \boldsymbol{B}_{i}\right\|_{F}^{2}-\left\|\boldsymbol{X}^{T} \boldsymbol{B}_{j}\right\|_{F}^{2}+\left\|\boldsymbol{B}_{j}^{T} \boldsymbol{B}_{i}\right\|_{F}^{2} .
\end{aligned}
$$

Then, the minimum in (18) may be found by solving $\mathbb{B} \hat{\boldsymbol{y}}=\mathbf{1}$, and then rescaling $\hat{\boldsymbol{y}}$ so that it sums to one. Algorithm 3 provides the pseudo-code for performing Grassmann Locality-constrained Coding (gLC). Fig. 3 illustrates the gLC algorithm conceptually.

A similar formulation albeit intrinsic, for the purpose of nonlinear embedding of Riemannian manifolds is developed by [23]. Aside from the different purpose (coding versus embedding), gLC can exploit an additional codebook learning step (as explained in $\S 6$ ) while dictionary learning based on the intrinsic formulation has no analytic solution.

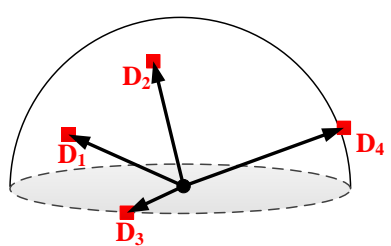

(a)

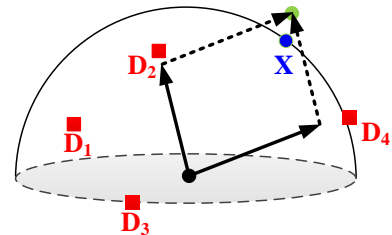

(b)

Figure 3: A conceptual diagram of the extrinsic locality constrained coding on Grassmann manifolds. The hemisphere is being used to represent $\mathcal{P G}(p, d)$. Each point on the surface of the hemisphere is intended to be a Grassmann point represented by a symmetric, idempotent and rank $p$ matrix. (a) A Grassmann dictionary on $\mathcal{P G}(p, d)$ with four atoms (red squares). (b) Locality constrained coding to describe a query point $\boldsymbol{X} \in \mathcal{P} \mathcal{G}(p, d)$. Here, only the closest atoms to the query point contribute in coding. With enough neighbors, it is possible to get arbitrarily close to the manifold. 


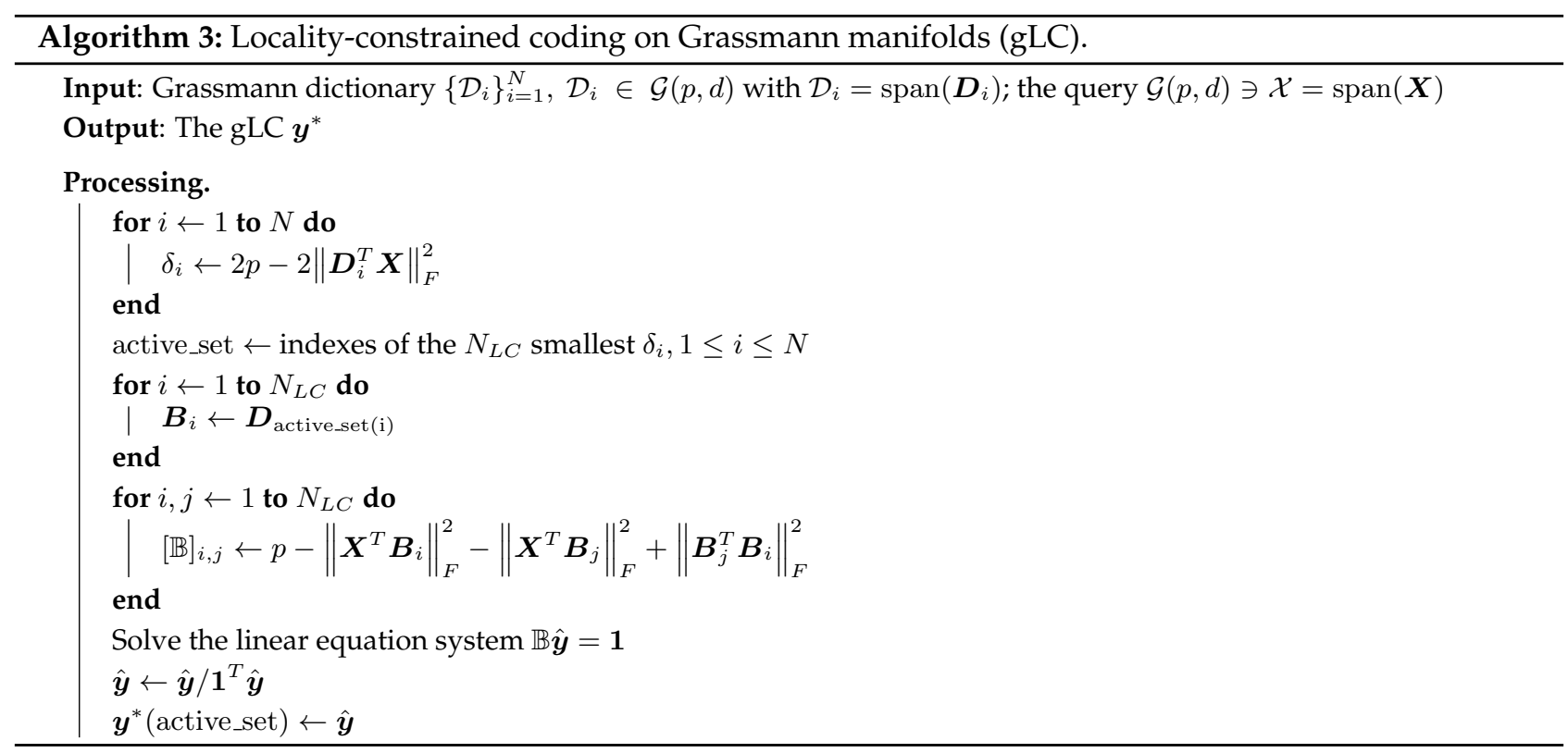

\subsection{Classification Based on Coding}

If the atoms in the dictionary are not labeled (e.g., if $\mathbb{D}$ is a generic dictionary not tied to any particular class), the generated sparse codes (vectors) for both training and query data can be fed to Euclidean-based classifiers like support vector machines [58] for classification. Inspired by the Sparse Representation Classifier (SRC) [70], when the atoms in sparse dictionary $\mathbb{D}$ are labeled, the generated codes of the query sample can be directly used for classification. In doing so, let

$$
\boldsymbol{y}_{c}=\left(\begin{array}{c}
{[\boldsymbol{y}]_{0} \delta\left(l_{0}-c\right)} \\
{[\boldsymbol{y}]_{1} \delta\left(l_{1}-c\right)} \\
\vdots \\
{[\boldsymbol{y}]_{N} \delta\left(l_{N}-c\right)}
\end{array}\right)
$$

be the class-specific sparse codes, where $l_{j}$ is the class label of atom $\mathcal{G}(p, d) \ni \mathcal{D}_{j}=\operatorname{span}\left(\boldsymbol{D}_{j}\right)$ and $\delta(x)$ is the discrete Dirac function. An efficient way of utilizing class-specific sparse codes is through computing residual errors. In this case, the residual error of query sample $\mathcal{G}(p, d) \ni \mathcal{X}=\operatorname{span}(\boldsymbol{X})$ for class $c$ is defined as:

$$
\varepsilon_{c}(\mathcal{X})=\left\|\widehat{\boldsymbol{X}}-\sum_{j=1}^{N}[\boldsymbol{y}]_{j} \widehat{\boldsymbol{D}}_{j} \delta\left(l_{j}-c\right)\right\|_{F}^{2} .
$$

Alternatively, the similarity between query sample $\mathcal{X}$ to class $c$ can be defined as $s(\mathcal{X}, c)=h\left(\boldsymbol{y}_{c}\right)$. The function $h(\cdot)$ could be a linear function like $\sum_{j=1}^{N}(\cdot)$ or even a non-linear one like max $(\cdot)$. Preliminary experiments suggest that Eq. (21) leads to higher classification accuracies when compared to the aforementioned alternatives.

\subsection{Extrinsic Nature of the Solution}

The solutions proposed in this section (e.g., (14)) apply to points in $\mathcal{P G}(p, d)$ and solve coding extrinsically, meaning $\sum_{j=1}^{N}[\boldsymbol{y}]_{j} \widehat{\boldsymbol{D}}_{j}$ is not necessarily a point on $\mathcal{G}(p, d)$. If, however, it is required that the linear combination of elements $\sum_{j}[\boldsymbol{y}]_{j} \widehat{\boldsymbol{D}}_{j}$ actually be used to represent a point on the manifold, then this can be found by projecting to the closest point on $\mathcal{P G}(p, d)$ using Theorem A.2 (see appendix). As shown there, the resulting point is the weighted chordal mean of the dictionary atoms $\widehat{\boldsymbol{D}}_{j}$ with coefficients $[\boldsymbol{y}]_{j}$.

Furthermore, the proposed methods follow the general principle of coding in that the over-completeness of $\mathbb{D}$ will approximate $\widehat{\boldsymbol{X}}$, and $\sum_{j}[\boldsymbol{y}]_{j} \widehat{\boldsymbol{D}}_{j}$ can be expected to be closely adjacent to a Grassmann point. An intrinsic version of (14) can be written as:

$$
l_{\mathcal{G}}(\mathcal{X}, \mathbb{D}) \triangleq \min _{\boldsymbol{y}}\left\|\widehat{\boldsymbol{X}}-\operatorname{Proj}\left(\sum_{j=1}^{N}[\boldsymbol{y}]_{j} \widehat{\boldsymbol{D}}_{j}\right)\right\|_{F}^{2}+\lambda\|\boldsymbol{y}\|_{1},
$$


where $\operatorname{Proj}\left(\sum_{j=1}^{N}[\boldsymbol{y}]_{j} \widehat{\boldsymbol{D}}_{j}\right)$ is the weighted chordal mean of the dictionary atoms, as shown by Theorem A.2. This formulation is precisely of the form (6), where the coding is the weighted chordal mean. The involvement of SVD makes solving (22) challenging. While seeking efficient ways of solving (22) is interesting, it is beyond the scope of this work. The coding error given by (22) and (14) will normally be very close, making (14) an efficient compromise solution.

\section{Dictionary Learning}

Given a finite set of observations $\mathbb{X}=\left\{\mathcal{X}_{i}\right\}_{i=1}^{m}, \mathcal{G}(p, d) \ni \mathcal{X}_{i}=\operatorname{span}\left(\boldsymbol{X}_{i}\right)$, the problem of dictionary learning on Grassmann manifolds is defined as minimizing the following cost function:

$$
h(\mathbb{D}) \triangleq \sum_{i=1}^{m} l_{\mathcal{G}}\left(\mathcal{X}_{i}, \mathbb{D}\right),
$$

with $\mathbb{D}=\left\{\mathcal{D}_{j}\right\}_{j=1}^{N}, \mathcal{G}(p, d) \ni \mathcal{D}_{j}=\operatorname{span}\left(\boldsymbol{D}_{j}\right)$ being a dictionary of size $N$. Here, $l_{\mathcal{G}}(\mathcal{X}, \mathbb{D})$ is a loss function and should be small if $\mathbb{D}$ is "good" at representing $\mathcal{X}$. In the following text, we elaborate on how a Grassmann dictionary can be learned.

Aiming for sparsity, the $\ell_{1}$-norm regularization is usually employed to obtain the most common form of $l_{\mathcal{G}}(\mathcal{X}, \mathbb{D})$ as depicted in Eq. (14). With this choice, the problem of dictionary learning on Grassmann manifolds can be written as:

$$
\min _{\left\{\boldsymbol{y}_{i}\right\}_{i=1}^{m}, \mathbb{D}} \sum_{i=1}^{m}\left\|\widehat{\boldsymbol{X}}_{i}-\sum_{j=1}^{N}\left[\boldsymbol{y}_{i}\right]_{j} \widehat{\boldsymbol{D}}_{j}\right\|_{F}^{2}+\lambda \sum_{i=1}^{m}\left\|\boldsymbol{y}_{i}\right\|_{1} .
$$

Due to the non-convexity of (24) and inspired by the solutions in Euclidean spaces, we propose to solve (24) by alternating between the two sets of variables, $\mathbb{D}$ and $\left\{\boldsymbol{y}_{i}\right\}_{i=1}^{m}$. More specifically, minimizing (24) over sparse codes $\boldsymbol{y}$ while dictionary $\mathbb{D}$ is fixed is a convex problem. Similarly, minimizing the overall problem over $\mathbb{D}$ with fixed $\left\{\boldsymbol{y}_{i}\right\}_{i=1}^{m}$ is convex as well.

Therefore, to update dictionary atoms we break the minimization problem into $N$ sub-minimization problems by independently updating each atom, $\widehat{\boldsymbol{D}}_{r}$, in line with general practice in dictionary learning [19]. To update $\widehat{\boldsymbol{D}}_{r}$, we write

$$
\sum_{i=1}^{m}\left\|\widehat{\boldsymbol{X}}_{i}-\sum_{j=1}^{N}\left[\boldsymbol{y}_{\boldsymbol{i}}\right]_{j} \widehat{\boldsymbol{D}}_{j}\right\|_{F}^{2}=\sum_{i=1}^{m}\left\|\left(\widehat{\boldsymbol{X}}_{i}-\sum_{j \neq r}\left[\boldsymbol{y}_{\boldsymbol{i}}\right]_{j} \widehat{\boldsymbol{D}}_{j}\right)-\left[\boldsymbol{y}_{\boldsymbol{i}}\right]_{r} \widehat{\boldsymbol{D}}_{r}\right\|_{F}^{2} .
$$

All other terms in (24) being independent of $\widehat{\boldsymbol{D}}_{r}$, and since $\left\|\widehat{\boldsymbol{D}}_{r}\right\|_{F}^{2}=p$ is fixed, minimizing this with respect to $\widehat{\boldsymbol{D}}_{r}$ is equivalent to minimizing $\mathcal{J}_{r}=-2\left\langle\boldsymbol{S}_{r}, \widehat{\boldsymbol{D}}_{r}\right\rangle$ where

$$
\boldsymbol{S}_{r}=\sum_{i=1}^{m}\left[\boldsymbol{y}_{\boldsymbol{i}}\right]_{r}\left(\widehat{\boldsymbol{X}}_{i}-\sum_{j \neq r}\left[\boldsymbol{y}_{\boldsymbol{i}}\right]_{j} \widehat{\boldsymbol{D}}_{j}\right) .
$$

Finally, minimizing $\mathcal{J}_{r}=-2\left\langle\boldsymbol{S}_{r}, \widehat{\boldsymbol{D}}_{r}\right\rangle$ is the same as minimizing $\left\|\boldsymbol{S}_{r}-\widehat{\boldsymbol{D}}_{r}\right\|$ over $\widehat{\boldsymbol{D}}_{r}$ in $\mathcal{P} \mathcal{G}(n, p)$. The solution to this problem is given by the $p$-leading eigenvectors of $\boldsymbol{S}_{r}$ according to Theorem A.1 in the appendix.

Algorithm 4 details the pseudo-code for learning a dictionary on Grassmann manifolds. Fig. 4 shows examples of a ballet dance and the atoms learned by the proposed method. From each atom, we plot the dominant eigendirection because it is visually more informative. Note that the learned atoms capture the ballerina movements.

To perform coding, we have relaxed the idempotent and rank constraints of the mapping $\Pi(\cdot)$ since matrix addition and subtraction do not preserve these constraints. However, for dictionary learning, the orthogonality constraint ensures the dictionary atoms have the required structure. Before concluding this section, we note that dictionary learning for gLC follows verbatim. The only difference from what we have developed in Algorithm 4 is the coding step which is done using Algorithm 3. 


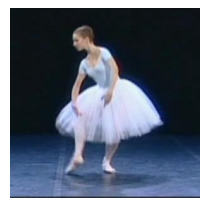

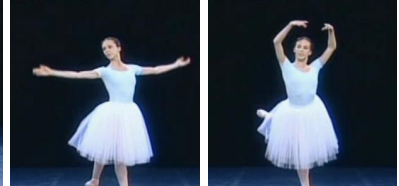

(a)
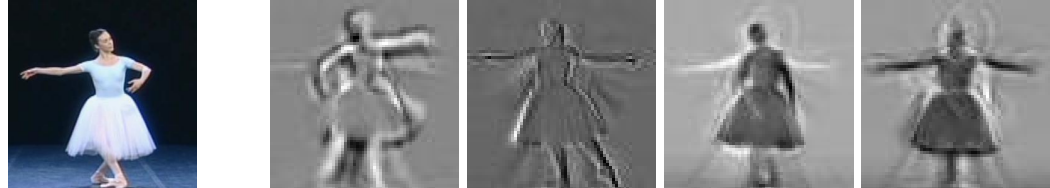

(b)

Figure 4: (a) Examples of actions performed by a ballerina. (b) The dominant eigenvectors for four atoms learned by the proposed Grassmann Dictionary Learning (gDL) method (grayscale images were used in gDL).

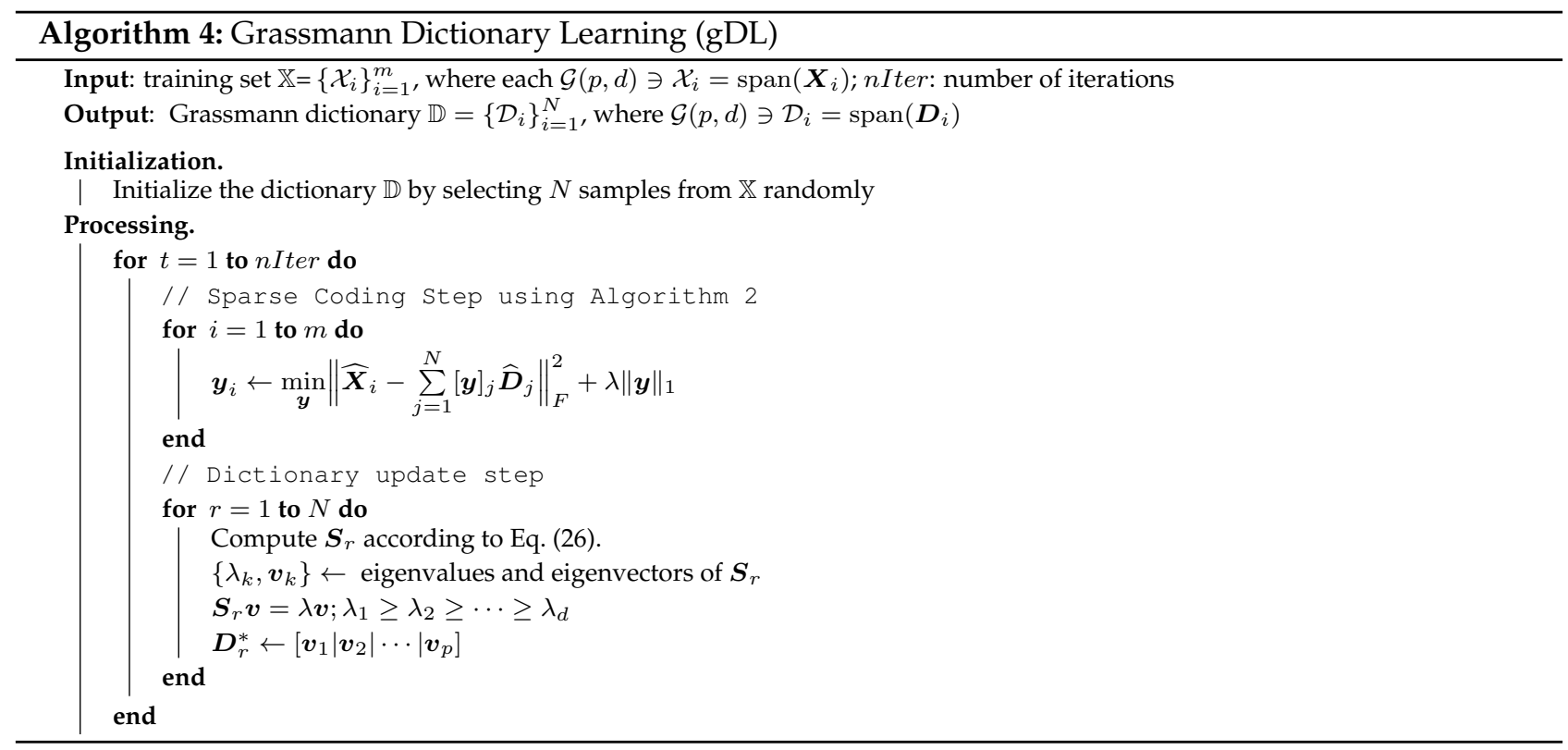

\section{Kernelized Coding and Dictionary Learning on Grassmann Manifolds}

In this section, we are interested in coding and dictionary learning on higher-dimensional (possibly infinite-dimensional) Grassmann manifolds. Such treatment can be helpful in dealing with non-linearity of data since one can hope higher-dimensional manifolds diminish non-linearity. This follows the practice of using higher dimensional spaces in vector spaces [58]. To this end, we make use of a mapping $\phi: \mathbf{R}^{d} \rightarrow \mathcal{H}$ from $\mathbf{R}^{d}$ into a Reproducing Kernel Hilbert Space (RKHS) $\mathcal{H}$ with a real-valued kernel function $k(\cdot, \cdot)$ on $\mathbf{R}^{d} \times \mathbf{R}^{d}$, such that $\forall \boldsymbol{x}, \boldsymbol{x}^{\prime} \in \mathbf{R}^{d},\left\langle\phi(\boldsymbol{x}), \phi\left(\boldsymbol{x}^{\prime}\right)\right\rangle=\phi(\boldsymbol{x})^{T} \phi\left(\boldsymbol{x}^{\prime}\right)=k\left(\boldsymbol{x}, \boldsymbol{x}^{\prime}\right)$ [58].

Our goal here is to perform both coding and dictionary learning in $\mathcal{H}$, but for efficiency we want to avoid explicitly working in $\mathcal{H}$. In other words, we would like to obtain the solutions by only using $k(\cdot, \cdot)$. In the following text we show how this can be achieved.

\subsection{Kernel Coding}

Let $\Psi(\boldsymbol{X})=\left[\boldsymbol{\psi}_{1}\left|\boldsymbol{\psi}_{2}\right| \cdots \mid \boldsymbol{\psi}_{p}\right]$ be an orthonormal basis of order $p$ for the column space of $\Phi(\boldsymbol{X})=$ $\left[\phi\left(\boldsymbol{x}_{1}\right)\left|\phi\left(\boldsymbol{x}_{2}\right)\right| \cdots \mid \phi\left(\boldsymbol{x}_{q}\right)\right], p \leq q$ in $\mathcal{H}$. The $q \times q$ Gram matrix $\Phi(\boldsymbol{X})^{T} \Phi(\boldsymbol{X})$ whose $i$-th row and $j$-th column entry is $k\left(\boldsymbol{x}_{i}, \boldsymbol{x}_{j}\right)$ can be decomposed as:

$$
\Phi(\boldsymbol{X})^{T} \Phi(\boldsymbol{X})=\boldsymbol{U}_{\boldsymbol{X}} \boldsymbol{\Sigma}_{\boldsymbol{X}} \boldsymbol{U}_{\boldsymbol{X}}^{T}
$$

The connection between $\boldsymbol{U}_{\boldsymbol{X}}$ and $\boldsymbol{\Psi}_{\boldsymbol{X}}$ is a "trick" used to compute the principal components of a matrix that has considerably less columns than rows [64], and can be easily established by picking the $p$ largest singular values of $\boldsymbol{\Sigma}_{\boldsymbol{X}}$ and corresponding elements of $\boldsymbol{U}_{\boldsymbol{X}}$ (denoted by $\downarrow$ below) as:

$$
\Psi(\boldsymbol{X})=\boldsymbol{\Phi}_{\boldsymbol{X}} \boldsymbol{U}_{\boldsymbol{X} \downarrow} \boldsymbol{\Sigma}_{\boldsymbol{X} \downarrow}^{-1 / 2}
$$

The sparse coding problem on a Grassmann manifold embedded in $\mathcal{H}$ can be understood as the kernel version of (14):

$$
\min _{\boldsymbol{y}}\left\|\widehat{\mathbf{\Psi}}(\boldsymbol{X})-\sum_{j=1}^{N}[\boldsymbol{y}]_{j} \widehat{\mathbf{\Psi}}\left(\boldsymbol{D}_{j}\right)\right\|_{F}^{2}+\lambda\|\boldsymbol{y}\|_{1} .
$$


A similar statement to what we have in $\S 5.2$ holds here for the convexity of (29). Therefore, sparse codes can be obtained if the Frobenius inner products between $\Psi(\boldsymbol{X})$ and elements of the dictionary, i.e., $\left\{\Psi\left(\boldsymbol{D}_{i}\right)\right\}_{i=1}^{N}$ are known. Given $\left\{\boldsymbol{z}_{i}\right\}_{i=1}^{q_{\boldsymbol{Z}}}, \boldsymbol{z}_{i} \in \mathbf{R}^{d}$ and $\left\{\boldsymbol{x}_{i}\right\}_{i=1}^{q_{\boldsymbol{X}}}, \boldsymbol{x}_{i} \in \mathbf{R}^{d}$, the Frobenius inner product between the corresponding subspaces $\Psi(\boldsymbol{Z})$ and $\Psi(\boldsymbol{X})$ in $\mathcal{H}$ can be obtained as:

$$
\begin{aligned}
\langle\Psi(\boldsymbol{Z}), \Psi(\boldsymbol{X})\rangle & =\operatorname{Tr}\left(\boldsymbol{\Psi}_{\boldsymbol{Z}}^{T} \boldsymbol{\Psi}_{\boldsymbol{X}}\right) \\
& =\operatorname{Tr}\left(\boldsymbol{\Sigma}_{\boldsymbol{Z} \downarrow}^{-1 / 2} \boldsymbol{U}_{\boldsymbol{Z} \downarrow}^{T} \Phi(\boldsymbol{Z})^{T} \Phi(\boldsymbol{X}) \boldsymbol{U}_{\boldsymbol{X} \downarrow} \boldsymbol{\Sigma}_{\boldsymbol{X} \downarrow}^{-1 / 2}\right) \\
& =\operatorname{Tr}\left(\boldsymbol{\Sigma}_{\boldsymbol{Z} \downarrow}^{-1 / 2} \boldsymbol{U}_{\boldsymbol{Z} \downarrow}^{T} \boldsymbol{K}(\boldsymbol{Z}, \boldsymbol{X}) \boldsymbol{U}_{\boldsymbol{X} \downarrow} \boldsymbol{\Sigma}_{\boldsymbol{X} \downarrow}^{-1 / 2}\right),
\end{aligned}
$$

where $\boldsymbol{K}(\boldsymbol{Z}, \boldsymbol{X})$ is a $q_{\boldsymbol{Z}} \times q_{\boldsymbol{X}}$ matrix where $i$-th row and $j$-th column entry is $k\left(\boldsymbol{z}_{i}, \boldsymbol{x}_{j}\right)$. Therefore, a similar approach to $\S 5.2$ can be employed to obtain the sparse codes in (29). Algorithm 5 provides the pseudo-code for performing kernel sparse coding on Grassmann manifolds (kgSC).

Based on the development in $\S 5.3$, the kernel version of gLC algorithm on Grassmann manifolds or $\mathrm{kgLC}$ for short can be obtained by computing the elements of matrix $\mathbb{B}$ in $\mathcal{H}$, i.e., kernelizing Eq.(20). That is,

$$
\begin{aligned}
{\left[\mathbb{B}_{\mathcal{H}}\right]_{i, j}=p } & -\left\|\Psi(\boldsymbol{X})^{T} \Psi\left(\boldsymbol{B}_{i}\right)\right\|_{F}^{2}-\left\|\Psi(\boldsymbol{X})^{T} \Psi\left(\boldsymbol{B}_{j}\right)\right\|_{F}^{2}+\left\|\Psi\left(\boldsymbol{B}_{j}\right)^{T} \Psi\left(\boldsymbol{B}_{i}\right)\right\|_{F}^{2} \\
=p & -\left\|\boldsymbol{\Sigma}_{\boldsymbol{B}_{i} \downarrow}^{-1 / 2} \boldsymbol{U}_{\boldsymbol{B}_{i \downarrow}}^{T} \boldsymbol{K}\left(\boldsymbol{B}_{i}, \boldsymbol{X}\right) \boldsymbol{U}_{\boldsymbol{X} \downarrow} \boldsymbol{\Sigma}_{\boldsymbol{X} \downarrow}^{-1 / 2}\right\|_{F}^{2} \\
& -\left\|\boldsymbol{\Sigma}_{\boldsymbol{B}_{j \downarrow}}^{-1 / 2} \boldsymbol{U}_{\boldsymbol{B}_{j} \downarrow}^{T} \boldsymbol{K}\left(\boldsymbol{B}_{j}, \boldsymbol{X}\right) \boldsymbol{U}_{\boldsymbol{X} \downarrow} \boldsymbol{\Sigma}_{\boldsymbol{X} \downarrow}^{-1 / 2}\right\|_{F}^{2} \\
& +\left\|\boldsymbol{\Sigma}_{\boldsymbol{B}_{i \downarrow} \downarrow}^{-1 / 2} \boldsymbol{U}_{\boldsymbol{B}_{i \downarrow}}^{T} \boldsymbol{K}\left(\boldsymbol{B}_{i}, \boldsymbol{B}_{j}\right) \boldsymbol{U}_{\boldsymbol{B}_{j \downarrow} \downarrow} \boldsymbol{\Sigma}_{\boldsymbol{B}_{j} \downarrow}^{-1 / 2}\right\|_{F}^{2} .
\end{aligned}
$$

Once we have $\mathbb{B}_{\mathcal{H}}$ at our disposal, the codes are obtained by first solving the linear system of equations, $\mathbb{B}_{\mathcal{H}} \hat{\boldsymbol{y}}=1$, and then rescaling the result to have unit $\ell_{1}$ norm.

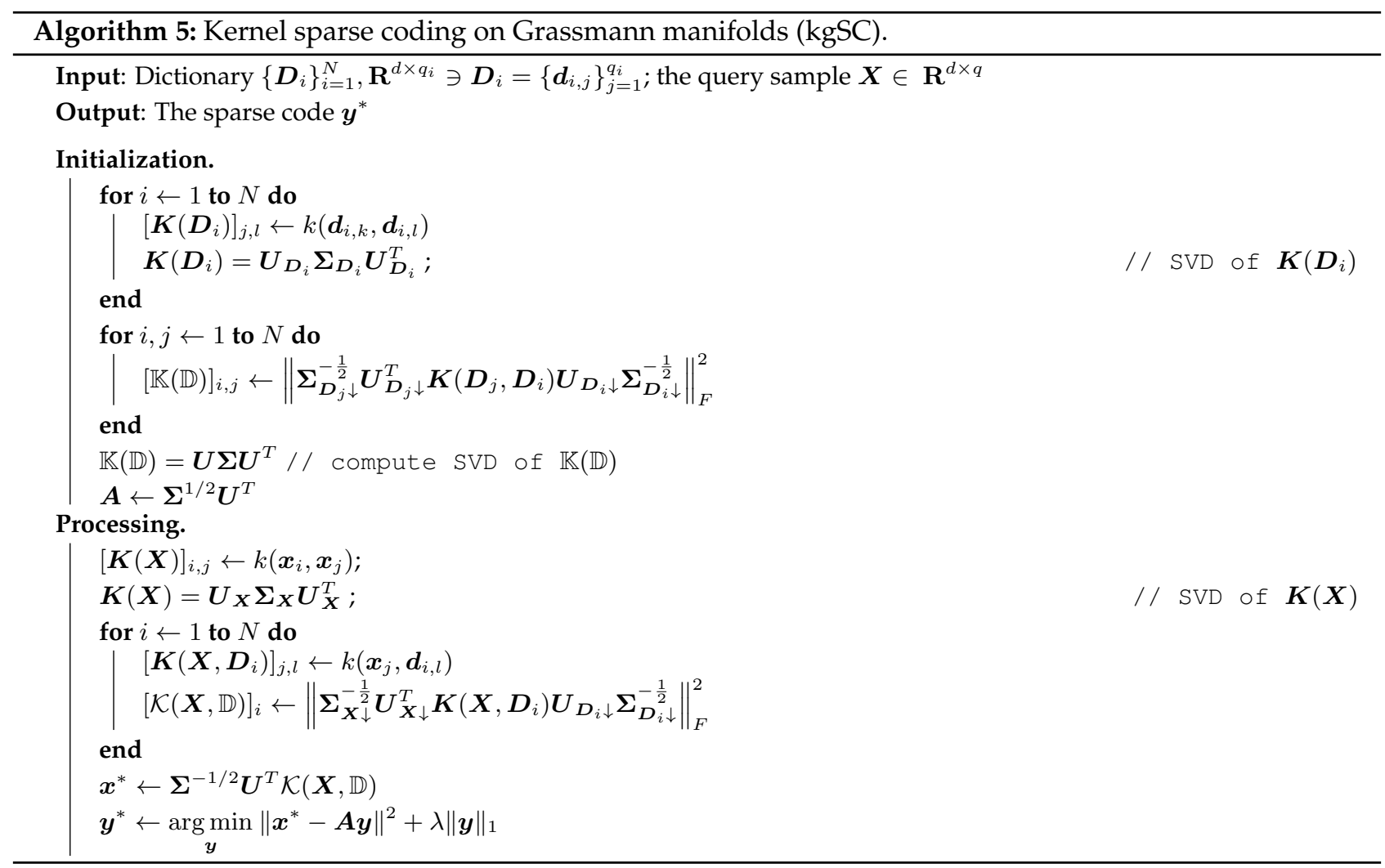




\subsection{Kernel Dictionary Learning}

Given a finite set of observations $\mathbb{X}=\left\{\boldsymbol{X}_{i}\right\}_{i=1}^{m}, \mathbf{R}^{d \times q_{i}} \ni \boldsymbol{X}_{i}=\left\{\boldsymbol{x}_{i, j}\right\}_{j=1}^{q_{i}}$, the problem of kernel dictionary learning in the RKHS $\mathcal{H}$ can be written by kernelizing (24) as:

$$
\min _{\left\{\boldsymbol{y}_{i}\right\}_{i=1, \mathbb{D}}^{m}} \sum_{i=1}^{m}\left\|\widehat{\boldsymbol{\Psi}}\left(\boldsymbol{X}_{i}\right)-\sum_{j=1}^{N}\left[\boldsymbol{y}_{i}\right]_{j} \widehat{\boldsymbol{\Psi}}\left(\boldsymbol{D}_{j}\right)\right\|_{F}^{2}+\lambda \sum_{i=1}^{m}\left\|\boldsymbol{y}_{i}\right\|_{1}
$$

Similar to the linear case, the dictionary in $\mathcal{H}$ is updated atom by atom (i.e., atoms are assumed to be independent) by fixing the codes $\left\{\boldsymbol{y}_{i}\right\}_{i=1}^{m}$. First, we note that a basis in $\mathcal{H}$ can be written as a linear combination of its samples. For $\Psi(\boldsymbol{X})$ and as shown in Eq. (28), $\Psi(\boldsymbol{X})=\Phi(\boldsymbol{X}) \boldsymbol{A}_{\boldsymbol{X}}$, where $\boldsymbol{A}_{\boldsymbol{X}}$ is obtained from $\boldsymbol{K}(\boldsymbol{X}, \boldsymbol{X})$. Similarly,

$$
\Psi\left(\boldsymbol{D}_{r}\right)=\Phi\left(\boldsymbol{D}_{r}\right) \boldsymbol{A}_{r}=\Phi\left(\bigcup_{i} \boldsymbol{X}_{i}\right) \boldsymbol{A}_{r},\left[\boldsymbol{y}_{i}\right]_{r} \neq 0
$$

As such, $\Psi\left(\boldsymbol{D}_{r}\right)$ is fully determined if $\boldsymbol{A}_{r}$ is known as $\boldsymbol{K}\left(\cdot, \boldsymbol{D}_{r}\right)=\boldsymbol{K}\left(\cdot, \bigcup_{i} \boldsymbol{X}_{i}\right),\left[\boldsymbol{y}_{i}\right]_{r} \neq 0$. The orthogonality constraint for $\Psi\left(\boldsymbol{D}_{r}\right)$ can be written as:

$$
\Psi\left(\boldsymbol{D}_{r}\right)^{T} \Psi\left(\boldsymbol{D}_{r}\right)=\boldsymbol{A}_{r}^{T} \boldsymbol{K}\left(\boldsymbol{D}_{r}, \boldsymbol{D}_{r}\right) \boldsymbol{A}_{r}=\mathbf{I}_{p},
$$

where $\boldsymbol{K}\left(\boldsymbol{D}_{r}, \boldsymbol{D}_{r}\right)=\boldsymbol{K}\left(\bigcup_{i} \boldsymbol{X}_{i}, \bigcup_{i} \boldsymbol{X}_{i}\right),\left[\boldsymbol{y}_{i}\right]_{r} \neq 0$. Following similar steps to what developed in $\S 6$, to obtain $\Psi\left(\boldsymbol{D}_{r}\right)$ we need to maximize $\operatorname{Tr}\left(\Psi\left(\boldsymbol{D}_{r}\right)^{T} \Gamma \Psi\left(\boldsymbol{D}_{r}\right)\right)$ by taking the orthogonality constraint (i.e., Eq. (34)) into account. Here $\Gamma$ is the kernel form of (26) written as:

$$
\Gamma=\sum_{i=1}^{m}\left[\boldsymbol{y}_{\boldsymbol{i}}\right]_{r}\left(\widehat{\mathbf{\Psi}}\left(X_{i}\right)-\sum_{j \neq r}\left[\boldsymbol{y}_{\boldsymbol{i}}\right]_{j} \widehat{\mathbf{\Psi}}\left(D_{j}\right)\right) .
$$

Defining

$$
\boldsymbol{B}(\boldsymbol{X}, \boldsymbol{Z})=\boldsymbol{K}(\boldsymbol{X}, \boldsymbol{Z}) \boldsymbol{A}_{\mathbf{Z}} \boldsymbol{A}_{\mathbf{Z}}^{T} \boldsymbol{K}(\boldsymbol{Z}, \boldsymbol{X}),
$$

and

$$
\boldsymbol{S}_{r}^{\Psi}=\sum_{i=1}^{m}\left[\boldsymbol{y}_{i}\right]_{r}\left(\boldsymbol{B}\left(\boldsymbol{D}_{r}, \boldsymbol{X}_{i}\right)-\sum_{\substack{j=1 \\ j \neq r}}^{N}\left[\boldsymbol{y}_{i}\right]_{j} \boldsymbol{B}\left(\boldsymbol{D}_{r}, \boldsymbol{D}_{j}\right)\right),
$$

maximizing $\operatorname{Tr}\left(\Psi\left(\boldsymbol{D}_{r}\right)^{T} \Gamma \Psi\left(\boldsymbol{D}_{r}\right)\right)$ with the orthogonality constraint boils down to:

$$
\begin{aligned}
\boldsymbol{A}_{r}^{*}=\underset{\boldsymbol{A}_{r}}{\operatorname{argmax}} \operatorname{Tr}\left(\boldsymbol{A}_{r}^{T} \boldsymbol{S}_{r}^{\Psi} \boldsymbol{A}_{r}\right), \\
\text { s.t. } \boldsymbol{A}_{r}^{T} \boldsymbol{K}\left(\boldsymbol{D}_{r}, \boldsymbol{D}_{r}\right) \boldsymbol{A}_{r}=\mathbf{I}_{p} .
\end{aligned}
$$

The solution of the above problem is given by the leading eigenvectors of the generalized eigenvalue problem $\boldsymbol{S}_{r}^{\Psi} \boldsymbol{v}=\lambda \boldsymbol{K}\left(\boldsymbol{D}_{r}, \boldsymbol{D}_{r}\right) \boldsymbol{v}$ [41]. In practice one might want to pick a small number of $\boldsymbol{X}_{i}$ that contributed more dominantly to Eq. (33) to describe $\Psi\left(\boldsymbol{D}_{r}\right)$ and hence reduce the computational load of dictionary learning. The steps of determining the kernel dictionary for Grassmann manifolds (kgDL) are shown in Algorithm 6. 


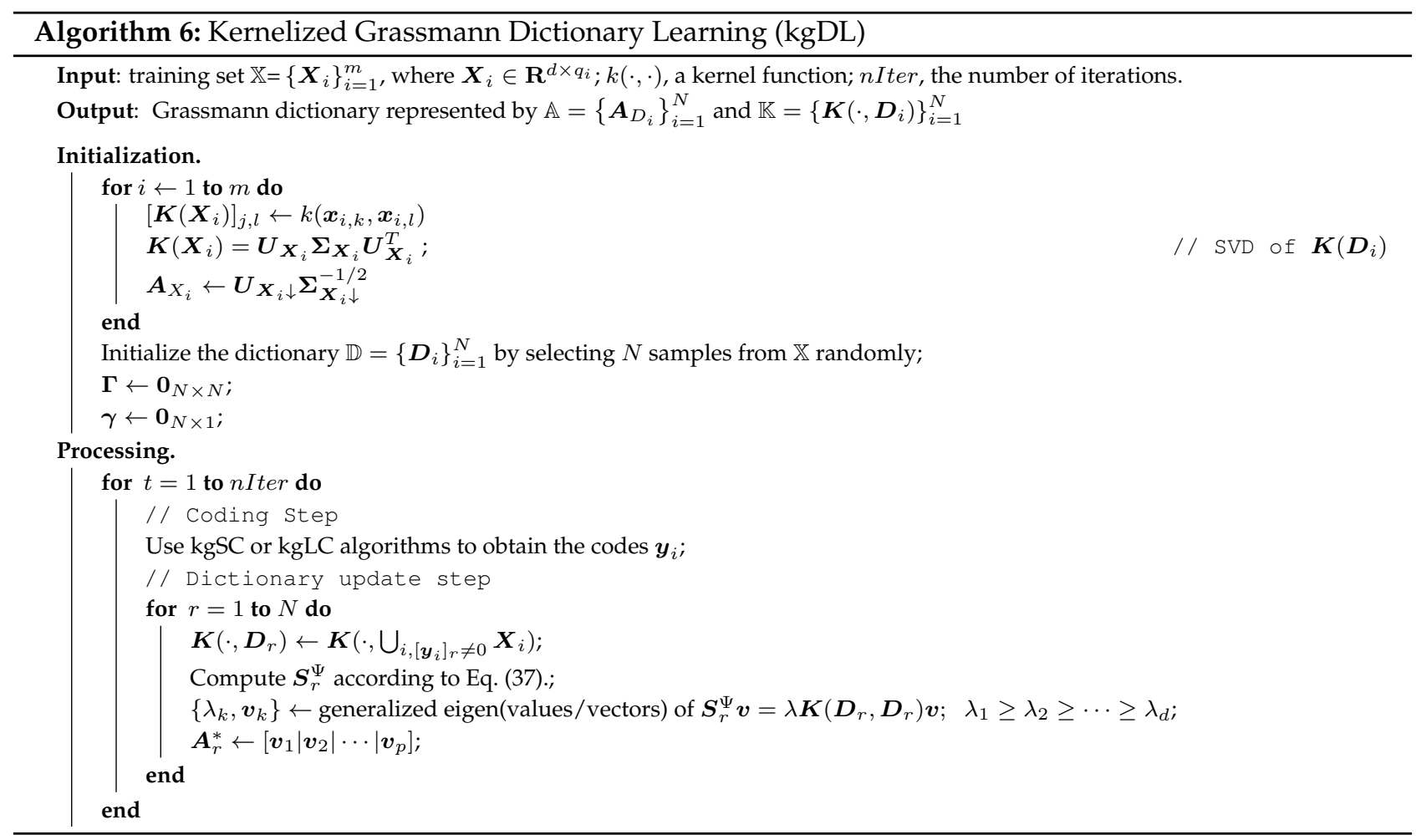

\section{Experiments}

Two sets of experiments ${ }^{3}$ are presented in this section. In the first set of experiments, we evaluate the performance of the proposed coding methods (as described in $\S 5$ and $\S 7$ ) without dictionary learning. This is to contrast proposed coding schemes to previous state-of-the-art methods on several popular closed-set classification tasks. To this end, each point in the training set is considered as an atom in the dictionary. Since the atoms in the dictionary are labeled in this case, the residual error approach for classification (as described in $\S 5.4$ and $\S 7$ ) will be used to determine the label of a query point. In the second set of experiments, the performance of the coding methods is evaluated in conjunction with the proposed dictionary learning algorithms described in $\S 6$. Before delving into experiments, we discuss how videos and image-sets can be modeled by linear subspaces and hence as points on Grassmann manifolds.

\subsection{Representing Image-Sets and Videos on Grassmann Manifolds}

Let us define a video as an ordered collection of images with time-stamp information, and an image-set as simply an orderless collection of images. In this section we briefly demonstrate how videos and image-sets can be modeled by subspaces (and hence as points on Grassmann manifolds). We first consider an approach where the time-stamp information is ignored, followed by an approach where the dynamics of image sequences are taken into account.

\subsubsection{Modeling of Appearance}

The appearance of an image-set or video $\mathbb{F}=\left\{\boldsymbol{f}_{1}, \boldsymbol{f}_{2}, \cdots, \boldsymbol{f}_{\tau}\right\}$, where $\boldsymbol{f}_{i} \in \mathbf{R}^{d}$ is the vectorized representation of $i$-th observation (frame in video), can be represented by a linear subspace through any orthogonalization procedure like SVD. More specifically, let $\boldsymbol{U} \boldsymbol{\Sigma} \boldsymbol{V}^{T}$ be the SVD of $\mathbb{F}$. The first $p$ columns of $\boldsymbol{U}$ represent an optimized subspace of order $p$ (in the mean square sense) for $\mathbb{F}$ and can be seen as a point on the Grassmann manifold $\mathcal{G}(p, d)$.

Modeling by linear subspaces generally does not take into account the order of images. While this property sounds restrictive, in many practical situations (like object recognition from video), the order of frames may not be important for decision making. However, it is possible to capture information related to order through an extended type of image-sets, obtained through a block Hankel matrix formalism [43].

\footnotetext{
${ }^{3}$ Matlab codes are available at https://sites.google.com/site/mehrtashharandi/
} 


\subsubsection{Modeling of Dynamics}

A video can be represented by an ARMA model to explicitly capture dynamics [18, 63]. A set of ordered images $\{\boldsymbol{f}(t)\}_{t=1}^{\tau} ; \boldsymbol{f}(t) \in \mathbf{R}^{d}$ can be modeled as the output of an ARMA model by:

$$
\begin{aligned}
\boldsymbol{f}(t) & =\boldsymbol{C} \boldsymbol{z}(t)+\boldsymbol{w}(t), & \boldsymbol{w}(t) & \sim \mathcal{N}(0, \boldsymbol{R}) . \\
\boldsymbol{z}(t+1) & =\boldsymbol{A} \boldsymbol{z}(t)+\boldsymbol{v}(t), & \boldsymbol{v}(t) & \sim \mathcal{N}(0, \boldsymbol{Q}),
\end{aligned}
$$

where $\boldsymbol{z}(t) \in \mathbf{R}^{n}$ is the hidden state vector at time $t, \boldsymbol{A} \in \mathbf{R}^{n \times n}$ and $\boldsymbol{C} \in \mathbf{R}^{d \times n}$ are the transition and measurement matrices, respectively, while $\boldsymbol{w}$ and $\boldsymbol{v}$ are noise components modeled as normal distributions with zero mean and covariance matrices $\boldsymbol{R} \in \mathbf{R}^{d \times d}$ and $\boldsymbol{Q} \in \mathbf{R}^{n \times n}$, respectively. Loosely speaking, one advantage of the ARMA model is that it decouples the appearance of the spatio-temporal data (modeled by $C$ ) from the dynamics (represented by $\boldsymbol{A}$ ).

The transition and measurement matrices can be estimated through a set of feature vectors. More specifically, if $\mathbb{F}_{\tau}=[\boldsymbol{f}(1)|\boldsymbol{f}(2)| \cdots \mid \boldsymbol{f}(\tau)]$ represents the feature matrix for time indexes $1,2, \cdots, \tau$, the estimated transition $\widehat{A}$ and measurement $\widehat{C}$ matrices can be obtained via the SVD of $\mathbb{F}_{\tau}=\boldsymbol{U} \boldsymbol{\Sigma} \boldsymbol{V}^{T}$, as follows:

$$
\begin{aligned}
\widehat{\boldsymbol{A}} & =\boldsymbol{\Sigma} \boldsymbol{V}^{T} \boldsymbol{D}_{1} \boldsymbol{V}\left(\boldsymbol{V}^{T} \boldsymbol{D}_{2} \boldsymbol{V}\right)^{-1} \boldsymbol{\Sigma}^{-1} . \\
\widehat{\boldsymbol{C}} & =\boldsymbol{U}
\end{aligned}
$$

where

$$
\boldsymbol{D}_{1}=\left[\begin{array}{cc}
\mathbf{0}_{\tau-1}^{T} & 0 \\
\mathbf{I}_{\tau-1} & \mathbf{0}_{\tau-1}
\end{array}\right] \text { and } \boldsymbol{D}_{2}=\left[\begin{array}{cc}
\mathbf{I}_{\tau-1} & \mathbf{0}_{\tau-1} \\
\mathbf{0}_{\tau-1}^{T} & 0
\end{array}\right] .
$$

Two ARMA models can be compared based on the subspace angles between the column-spaces of their observability matrices [15]. The extended observability matrix of an ARMA model is given by

$$
\boldsymbol{O}_{\infty}=\left[\boldsymbol{C}^{T}\left|(\boldsymbol{C A})^{T}\right|\left(\boldsymbol{C A}^{\mathbf{2}}\right)^{T}|\cdots|\left(\boldsymbol{C} \boldsymbol{A}^{\boldsymbol{n}}\right)^{T} \mid \cdots\right]^{T} .
$$

The extended observability matrix is usually approximated by the finite observability matrix as [63]:

$$
\boldsymbol{O}_{m}=\left[\boldsymbol{C}^{T}\left|(\boldsymbol{C A})^{T}\right|\left(\boldsymbol{C} \boldsymbol{A}^{\mathbf{2}}\right)^{T}|\cdots|\left(\boldsymbol{C} \boldsymbol{A}^{(m-1)}\right)^{T}\right]^{T} .
$$

For a given video, the finite observability parameter of the ARMA model is estimated as described above. To represent the subspace spanned by the columns of $\boldsymbol{O}_{m}$, an orthonormal basis can be computed through Gram-Schmidt orthonormalization. As a result, a linear dynamic system can be described as a point on a Grassmann manifold corresponding to the column space of the observability matrix. The appearance modeling presented in $\S 8.1 .1$ can be seen as a special case of ARMA modeling, where $m=1$.

\subsection{Coding on Grassmann Manifolds}

In this part we compare and contrast the performance of the proposed methods against several state-of-the-art methods: Discriminant Canonical Correlation Analysis (DCC) [40], kernelized Affine Hull Method (KAHM) [11], Grassmann Discriminant Analysis (GDA) [30], Graph-embedding Grassmann Discriminant Analysis (GGDA) [33] and the intrinsic sparse coding (iSC) [36]. We evaluate the performance on the tasks of (i) gender recognition from gait, (ii) hand gesture recognition, and (iii) scene analysis.

DCC is an iterative learning method that maximizes a measure of discrimination between image sets where the distance between sets is expressed by canonical correlations. In KAHM, images are considered as points in a linear or affine feature space, while image sets are characterized by a convex geometric region (affine or convex hull) spanned by their feature points. GDA can be considered as an extension of kernel discriminant analysis over Grassmann manifolds [30]. In GDA, a transform over the Grassmann manifold is learned to simultaneously maximize a measure of inter-class distances and minimize intra-class distances. GGDA can be considered as an 
extension of GDA, where a local discriminant transform over Grassmann manifolds is learned. This is achieved by incorporating local similarities/dissimilarities through within-class and between-class similarity graphs.

We denote Grassmann sparse coding, Grassmann locality linear coding and their kernel extensions by $g S C$, $g L C, k g S C$ and $k g L C$, respectively. Based on preliminary experiments, the Gaussian kernel [58], defined as $k(\boldsymbol{a}, \boldsymbol{b})=\exp \left(-\gamma\|\boldsymbol{a}-\boldsymbol{b}\|^{2}\right)$, was used in $\mathrm{kgSC}$ and $\mathrm{kgLC}$. The value of the $\gamma$ parameter in all experiments was determined by cross validation.

In the following three experiments, the Grassmannian dictionary for the proposed gSC, gLC, $\mathrm{kgSC}$ and $\mathrm{kgLC}$ was constituted of all available training data. The classification method described in $\S 5.4$ was used to determine the label of a query sample. The class specific residual error in the case of $\mathrm{kgSC}$ and $\mathrm{kgLC}$ is obtained by kernelizing Eq. (21) as:

$$
\varepsilon_{c}(\mathcal{X})=\left\|\widehat{\Psi}_{\boldsymbol{X}}-\sum_{j=1}^{N}[\boldsymbol{y}]_{j} \widehat{\boldsymbol{\Psi}}_{\boldsymbol{D}_{j}} \delta(l(j)-c)\right\|_{F}^{2},
$$

where $l(j)$ is the class label of the $j^{\text {th }}$ atom and $\delta(x)$ is the discrete Dirac function.

\subsubsection{Gender Recognition from Gait}

Gait is defined as "manner of walking" and can be used as a biometric measure to recognize, among other things, the gender of humans [75]. For the task of gender recognition from gait data, we have used Dataset-B of the CASIA Gait Database [78] which constitutes of 124 individuals (93 males and 31 females). In the CASIA dataset, the gait of each subject has been captured from 11 angles. Every video is represented by one gait energy image (GEI) of size $32 \times 32$, which has been shown to be effective in recognition of gender [75]. Cropped samples of GEI images are shown in Fig. 5.

We used the videos captured with normal clothes and created a subspace of order 6 (based on preliminary experiments) using the corresponding 11 GEIs. This resulted in 731 points on $\mathcal{G}(1024,6)$. We then randomly selected 20 individuals (10 male, 10 female) as the training set and used the remaining individuals for testing. There is no overlap of individuals between the training and test sets.

Table 1 shows a comparison of gSC, gLC and their kernelized versions against DCC, KAHM, GDA and GGDA. All four proposed methods consistently outperform previous state-of-the-art algorithms with a big margin. The highest accuracy is attained by $\mathrm{kgSC}$, followed by $\mathrm{kgLC}$. As expected, the kernel extensions perform better than gSC and gLC. However, the burden of determining the kernel parameters could be sometimes overwhelming.

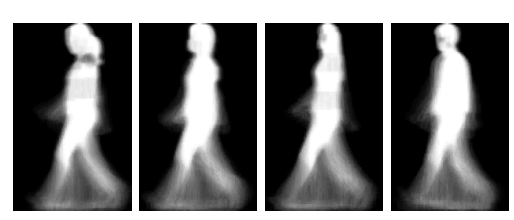

Figure 5: GEI samples from the CASIA gait dataset [78].

Table 1: Recognition rate on the CASIA dataset for KAHM [11], GDA [30], GGDA [33], iSC [36] and the proposed approaches.

\begin{tabular}{lc}
\hline Method & Accuracy \\
\hline DCC [40] & $85.9 \pm 6.6$ \\
KAHM [11] & $89.8 \pm 2.4$ \\
GDA [30] & $76.4 \pm 5.8$ \\
GGDA [33] & $84.3 \pm 4.8$ \\
iSC [36] & $86.9 \pm 3.2$ \\
\hline gSC & $\mathbf{9 4 . 3} \pm \mathbf{2 . 1}$ \\
gLC & $\mathbf{9 3 . 7} \pm \mathbf{2 . 1}$ \\
kgSC & $\mathbf{9 5 . 6} \pm \mathbf{2 . 1}$ \\
kgLC & $\mathbf{9 5 . 2} \pm \mathbf{1 . 6}$ \\
\hline
\end{tabular}




\subsubsection{Hand Gesture Recognition}

For the hand-gesture recognition task, we used the Cambridge hand-gesture dataset [39] which consists of 900 image sequences of 9 gesture classes. Each class has 100 image sequences performed by 2 subjects, captured under 5 illuminations and 10 arbitrary motions. The 9 classes are defined by three primitive hand shapes and three primitive motions. Each sequence was recorded at $30 \mathrm{fps}$ with a resolution of $320 \times 240$, in front of a fixed camera having roughly isolated gestures in space and time. See Fig. 6 for examples. We followed the test protocol defined by [39], and resized all sequences to $20 \times 20 \times 20$. Sequences with normal illumination are considered for training while the remaining sequences (with different illumination characteristics) are used for testing.

As per [39], we report the recognition rates for the four illumination sets. In addition to GDA, GGDA and KAHM, the proposed methods were also compared against Tensor Canonical Correlation Analysis (TCCA) [39] and Product Manifolds (PM) [44]. TCCA, as the name implies, is the extension of canonical correlation analysis to multiway data arrays or tensors. Canonical correlation analysis is a standard method for measuring the similarity between subspaces [39]. In the PM method a tensor is characterized as a point on a product manifold and classification is performed on this space. The product manifold is created by applying a modified high order singular value decomposition on the tensors and interpreting each factorized space as a Grassmann manifold.

For Grassmann-based methods, we represented each video through ARMA modeling. The observability order of the ARMA model ( $m$ in Eq. (43)) and the subspace dimension (order of matrix $C$ ) were selected as 5 and 10, respectively. The results, presented in Table 2, show that the proposed approaches obtain the highest performance. $\mathrm{kgLC}$ achieves the best recognition accuracy on all four sets. KAHM performs very poorly in this task, which we conjecture is due to the illumination differences between the training and test sets.
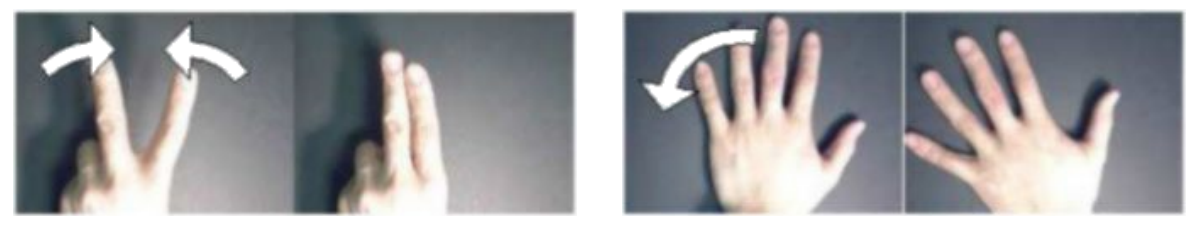

Figure 6: Examples of hand actions in the Cambridge dataset [39].

Table 2: Recognition accuracy for the hand-gesture recognition task using KAHM [11], GDA [30], GGDA [33], TCCA [39], Product Manifold (PM) [44], iSC [36] and the proposed approaches.

\begin{tabular}{lccccc}
\hline Method & Set1 & Set2 & Set3 & Set4 & Overall \\
\hline TCCA [39] & 81 & 81 & 78 & 86 & $82 \pm 3.5$ \\
KAHM [11] & 43 & 43 & 43 & 41 & $43 \pm 1.4$ \\
GDA [30] & 92 & 85 & 84 & 87 & $87.4 \pm 3.8$ \\
GGDA [33] & 91 & 91 & 88 & 94 & $91.1 \pm 2.5$ \\
PM [44] & 93 & 89 & 91 & 94 & $91.7 \pm 2.3$ \\
iSC [36] & 93 & 94 & 89 & 92 & $92.4 \pm 2.1$ \\
\hline gSC & $\mathbf{9 3}$ & $\mathbf{9 2}$ & $\mathbf{9 3}$ & $\mathbf{9 4}$ & $\mathbf{9 3 . 3} \pm \mathbf{0 . 9}$ \\
gLC & $\mathbf{9 6}$ & $\mathbf{9 4}$ & $\mathbf{9 6}$ & $\mathbf{9 7}$ & $\mathbf{9 5 . 4} \pm \mathbf{1 . 3}$ \\
kgSC & $\mathbf{9 6}$ & $\mathbf{9 2}$ & $\mathbf{9 3}$ & $\mathbf{9 7}$ & $\mathbf{9 4 . 4} \pm \mathbf{2 . 0}$ \\
kgLC & $\mathbf{9 6}$ & $\mathbf{9 4}$ & $\mathbf{9 6}$ & $\mathbf{9 8}$ & $\mathbf{9 5 . 7} \pm \mathbf{1 . 6}$ \\
\hline
\end{tabular}




\subsubsection{Scene Analysis}

For scene analysis, we employed the UCSD traffic dataset [12], which contains 254 video sequences of highway traffic of varying patterns (e.g. light, heavy) in various weather conditions (e.g., cloudy, raining, sunny). Each video was recorded with a resolution of $320 \times 240$ pixels, for a duration ranging from 42 to 52 frames. Here we have used a normalized grayscale $48 \times 48$ version of the dataset. The normalization process for each video clip involves subtracting the mean image and normalizing the pixel intensities to unit variance. This is useful to reduce the impact of illumination variations.

The dataset is labeled into three classes with respect to the amount of traffic congestion in each sequence. In total there are 44 sequences of heavy traffic (slow or stop-and-go speeds), 45 of medium traffic (reduced speed), and 165 of light traffic (normal speed). See Fig. 7 for examples.

We represented each video on a Grassmann manifold through ARMA modeling. The observability order of the ARMA model and the subspace dimension were selected as 5 and 10 respectively.

In addition to GDA, GGDA and KAHM, the proposed methods were also compared against Linear Dynamical System (LDS) and Compressive Sensing Linear Dynamical System (CS-LDS) [57]. The results, presented in Table 3, show that the proposed approaches obtain the best overall performance, with $\mathrm{kgLC}$ achieving the highest overall accuracy. It is worth mentioning that the performance of $\mathrm{kgLC}$ competes with the state-of-the-art algorithms on this dataset (e.g., Ravichandran et al. report an accuracy of 95.6\% [54]).
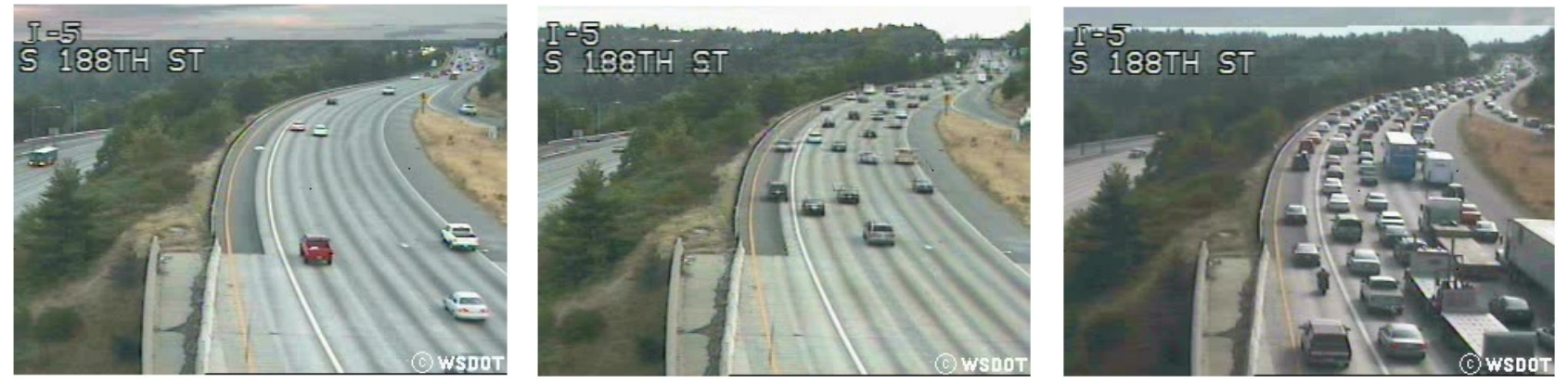

Figure 7: Representative examples of the three classes in UCSD traffic video dataset [12]. From left to right: examples of light, medium, and heavy traffic.

Table 3: Average correct recognition rate on the UCSD video traffic dataset for dynamic spatio-temporal models using LDS [57], Compressive-Sensing LDS [57], GDA [30], GGDA [33], and the proposed approaches.

\begin{tabular}{lccccc}
\hline Method & Exp1 & Exp2 & Exp3 & Exp4 & Overall \\
\hline LDS [57] & 85.7 & 85.9 & 87.5 & 92.1 & $87.8 \pm 3.0$ \\
CS-LDS [57] & 84.1 & 87.5 & 89.1 & 85.7 & $86.6 \pm 2.2$ \\
KAHM [11] & 84.1 & 79.7 & 82.8 & 84.1 & $82.7 \pm 2.1$ \\
GDA [30] & 82.5 & 85.9 & 70.3 & 77.8 & $79.1 \pm 6.7$ \\
GGDA [33] & 87.3 & 89.1 & 90.6 & 90.5 & $89.4 \pm 1.5$ \\
iSC [36] & 93.7 & 87.5 & 90.6 & 96.8 & $92.2 \pm 4.0$ \\
\hline gSC & $\mathbf{9 3 . 7}$ & $\mathbf{8 7 . 5}$ & $\mathbf{9 5 . 3}$ & $\mathbf{9 5 . 2}$ & $\mathbf{9 2 . 9} \pm \mathbf{3 . 7}$ \\
gLC & $\mathbf{9 6 . 8}$ & $\mathbf{8 5 . 9}$ & $\mathbf{9 2 . 2}$ & $\mathbf{9 3 . 7}$ & $\mathbf{9 2 . 2} \pm \mathbf{4 . 5}$ \\
kgSC & $\mathbf{9 6 . 8}$ & $\mathbf{8 9 . 1}$ & $\mathbf{9 5 . 3}$ & $\mathbf{9 8 . 4}$ & $\mathbf{9 4 . 9} \pm \mathbf{4 . 1}$ \\
kgLC & $\mathbf{9 5 . 2}$ & $\mathbf{9 2 . 2}$ & $\mathbf{9 6 . 9}$ & $\mathbf{9 6 . 8}$ & $\mathbf{9 5 . 3} \pm \mathbf{2 . 2}$ \\
\hline
\end{tabular}




\subsection{Synthetic Data}

To contrast the Log-Euclidean (lE-SC) and intrinsic (iSC) [10,36] solutions against the proposed gSC approach we performed two experiments with synthetic data. Specifically, we considered two multi-class classification problems over $\mathcal{G}(2,6)$. The first experiment involved a relatively simple classification problem that matched the properties of the Log-Euclidean approach, while the second experiment considered a more realistic scenario.

In both experiments, we randomly generated four classes over the $\mathcal{G}(2,6)$, where the samples in each class obey a normal distribution on a specific tangent space of $\mathcal{G}(2,6)$. This can be achieved by considering normal distributions over the specific tangent space of $\mathcal{G}(2,6)$ followed by mapping the points back to $\mathcal{G}(2,6)$ using the exponential map (see [6] for details of the exponential map). We created four classification problems with increasing difficulty, by fixing the mean of each class and increasing the class variance. In the following discussion, the problems will be referred as 'easy', 'medium', 'hard', and 'very hard'.

For a given problem, 8 samples per class were considered as the dictionary atoms, while 1000 samples per class were generated as query data. This results in a multiclass recognition problem with 4000 samples and a dictionary of size 32. All the generated samples were then mapped back to the manifold using the exponential map and were used in the Log-Euclidean, intrinsic and the proposed sparse coding approaches. For each task, the data generation procedure was repeated ten times; average recognition rates are reported.

In the first experiment, we considered distributions over the identity tangent space, i.e., $\mathcal{P}=\operatorname{span}\left(\left[\begin{array}{c}\mathbf{I}_{2} \\ \mathbf{0}\end{array}\right]\right)$. The results are presented in Table 4 under Experiment \#1. By increasing the class variance, samples from various classes are intertwined, which in turn leads to a decrease in recognition accuracy. As for the log-Euclidean approach, we considered two setups. In the first setup, the center of projection was fixed at $\mathcal{P}$. In the second setup (shown as IE-SC-adaptive), the center of projection was set to the Fréchet mean of data. Even though this experiment matches the characteristics of the Log-Euclidean approach (since the prior knowledge of class distribution is available), both gSC and iSC approaches obtain on par performance for the easy case. For the medium case, Log-Euclidean approaches achieve higher accuracy followed by gSC and iSC. We note that the fixed log-Euclidean method performs better than the adaptive setup for this experiment.

In the second experiment we relaxed the location of tangent space in order to simulate a more challenging scenario. More specifically, instead of generating distributions over the identity tangent space, the tangent space was selected randomly. As shown in Table 4 under Experiment \#2, the Log-Euclidean approaches perform poorly when compared to gSC and iSC. Among the two setups of the log-Euclidean approach, the adaptive one performs better than the fixed one. Similar to the previous experiments, the gSC approach consistently outperforms iSC.

Table 4: Comparison of the proposed gSC approach with the Log-Euclidean sparse coding (IE-SC) and intrinsic sparse coding (iSC) [36] methods on synthetic data. In the first experiment, samples in each class obey a normal distribution over the identity tangent space. The second experiment reflects a more challenging scenario where samples in each class obey a normal distribution over a random tangent space, instead of the identity tangent space.

\begin{tabular}{lcccc}
\hline & \multicolumn{2}{c}{ Experiment \#1 } & \multicolumn{2}{c}{ Experiment \#2 } \\
Task & Easy & Medium & Hard & Very Hard \\
\hline lE-SC & $99.5 \%$ & $90.8 \%$ & $55.7 \%$ & $49.9 \%$ \\
lE-SC-adaptive & $92.9 \%$ & $88.7 \%$ & $57.3 \%$ & $50.6 \%$ \\
iSC [36] & $98.6 \%$ & $84.1 \%$ & $64.7 \%$ & $53.4 \%$ \\
gSC & $99.2 \%$ & $86.6 \%$ & $66.9 \%$ & $57.4 \%$ \\
\hline
\end{tabular}




\subsection{Dictionary Learning}

Here we analyze the performance of the proposed dictionary learning techniques as described in $\S 6$ on three classification tasks: face recognition, action recognition and dynamic texture classification. In all the following experiments, an SVM classifier with a Gaussian kernel was used to perform recognition. That is, the training and testing data were first coded by the learned dictionary and then the sparse codes were fed to an SVM classifier. Parameters for the SVM classifier were determined by cross validation.

Since the intrinsic dictionary learning as proposed by [36] has no analytic solution on Grassmann manifolds, we will just compare gSC, gLC and their kernel extensions in conjunction with dictionary learning against Discriminant Canonical Correlation Analysis (DCC) [40], kernelized Affine Hull Method (KAHM) [11], Grassmann Discriminant Analysis (GDA) [30] and Graph-embedding Grassmann Discriminant Analysis (GGDA) [33] in the following experiments.

\subsubsection{Face Recognition}

While face recognition from a single still image has been extensively studied, recognition based on a group of still images is relatively new. A popular choice for modeling image-sets is by representing them through linear subspaces [30,33]. For the task of image-set face recognition, we used the YouTube celebrity dataset [38] which contains 1910 video clips of 47 subjects. See Fig. 8 for examples. Face recognition on this dataset is challenging, since the videos have a high compression ratio and most of them have low-resolution.

To create an image set from a video, we used a cascaded face locator [66] to extract face regions from each video, followed by resizing regions to $96 \times 96$ and describing them via histogram of Local Binary Patterns (LBP) [50]. Then each image set (corresponding to a video) was represented by a linear subspace of order 5 . We randomly chose $70 \%$ of the dataset for training and the remaining $30 \%$ for testing. The process of random splitting was repeated ten times and the average classification accuracy is reported.

The results in Table 5 show that the proposed coding methods (using dictionaries provided by their corresponding dictionary learning algorithms) outperform the competitors. $\mathrm{kgSC}$ with dictionary learning achieved the highest accuracy of $73.91 \%$, more than 3 percentage points better than gSC with dictionary learning. Similarly, the performance of kgLC with dictionary learning is observed to be higher than gLC with dictionary learning.
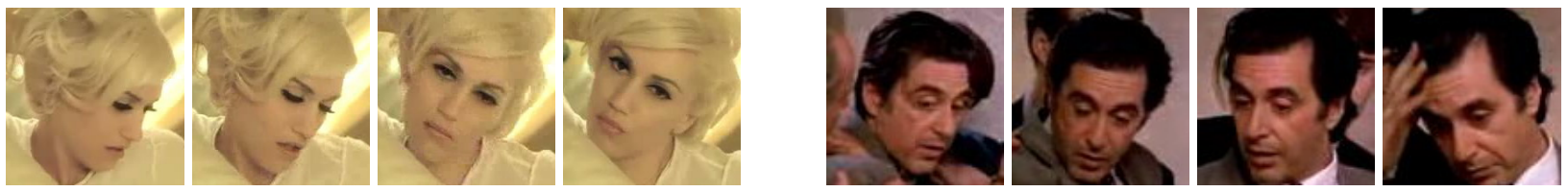

Figure 8: Examples from the YouTube celebrity dataset (grayscale versions of images were used in experiments).

Table 5: Average correct recognition rate (CRR) on the YouTube celebrity dataset.

\begin{tabular}{lc}
\hline Method & CRR \\
\hline DCC [40] & $60.21 \pm 2.9$ \\
KAHM [11] & $67.49 \pm 3.5$ \\
GDA [30] & $58.72 \pm 3.0$ \\
GGDA [33] & $61.06 \pm 2.2$ \\
\hline gSC-dic & $\mathbf{7 0 . 4 7} \pm \mathbf{1 . 7}$ \\
gLC-dic & $\mathbf{7 1 . 7 4} \pm \mathbf{2 . 3}$ \\
kgSC-dic & $\mathbf{7 3 . 9 1} \pm \mathbf{1 . 9}$ \\
kgLC-dic & $\mathbf{7 3 . 5 3} \pm \mathbf{2 . 3}$ \\
\hline
\end{tabular}




\subsubsection{Ballet Dataset}

The Ballet dataset contains 44 videos collected from an instructional ballet DVD [68]. The dataset consists of 8 complex motion patterns performed by 3 subjects, The actions include: 'left-to-right hand opening', 'right-to-left hand opening', 'standing hand opening', 'leg swinging', 'jumping', 'turning', 'hopping' and 'standing still'. Fig. 9 shows examples. The dataset is challenging due to the significant intra-class variations in terms of speed, spatial and temporal scale, clothing and movement.

We extracted 2400 image sets by grouping 6 frames that exhibited the same action into one image set. We described each image set by a subspace of order 4 with Histogram of Oriented Gradients (HOG) as frame descriptor [16]. Available samples were randomly split into training and testing sets (the number of image sets in both sets was even). The process of random splitting was repeated ten times and the average classification accuracy is reported.

Table 6 shows that all proposed coding approaches have superior performance as compared to DCC, KAHM, GDA and GGDA. For example, the difference between gSC with dictionary learning (gSC-dic) and the closest state-of-the-art competitor (GGDA), is more than six percentage points.
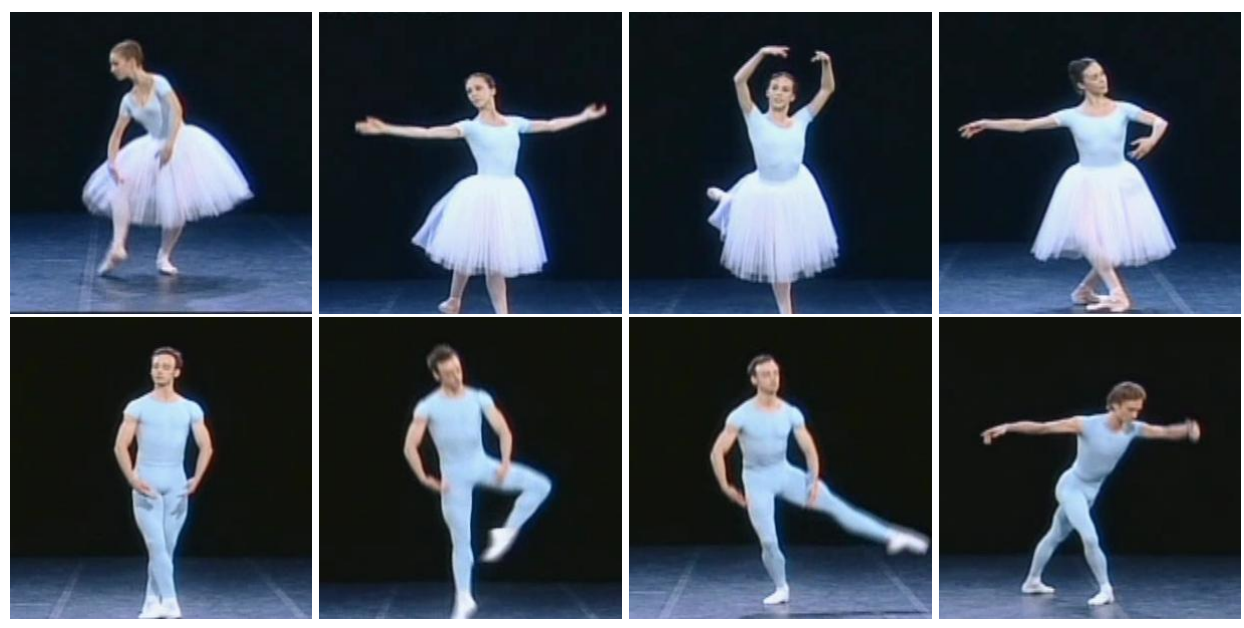

Figure 9: Examples from the Ballet dataset [68].

Table 6: Average recognition rate on the Ballet dataset.

\begin{tabular}{lc}
\hline Method & CRR \\
\hline DCC [40] & $41.95 \pm 9.6$ \\
KAHM [11] & $70.05 \pm 0.9$ \\
GDA [30] & $67.33 \pm 1.1$ \\
GGDA [33] & $73.54 \pm 2.0$ \\
\hline gSC-dic & $\mathbf{7 9 . 6 4} \pm \mathbf{1 . 1}$ \\
gLC-dic & $\mathbf{8 1 . 4 2} \pm \mathbf{0 . 8}$ \\
kgSC-dic & $\mathbf{8 3 . 5 3} \pm \mathbf{0 . 8}$ \\
kgLC-dic & $\mathbf{8 6 . 9 4} \pm \mathbf{1 . 1}$ \\
\hline
\end{tabular}




\subsubsection{Dynamic Texture Classification}

Dynamic textures are videos of moving scenes that exhibit certain stationary properties in the time domain [22, 71]. Such videos are pervasive in various environments, such as sequences of rivers, clouds, fire, swarms of birds, humans in crowds. In our experiment, we used the challenging DynTex++ dataset [22], which is comprised of 36 classes, each of which contains 100 sequences with a fixed size of $50 \times 50 \times 50$ (see Fig. 10 for example classes). We split the dataset into training and testing sets by randomly assigning half of the videos of each class to the training set and using the rest as query data. The random split was repeated twenty times; average accuracy is reported.

To generate Grassmann points, we used histogram of LBP from Three Orthogonal Planes (LBP-TOP) [77] which takes into account the dynamics within the videos. To this end, each video is split into subvideos of length 10, with a 7 frame overlap. Each subvideo is then described by a histogram of LBP-TOP features. From the subvideo descriptors, we extracted a subspace of order 5 as the video representation on a Grassmann manifold.

In addition to DCC, KAHM, GDA and GGDA, the proposed approaches were compared against two methods specifically designed for dynamic texture classification: dynamic fractal spectrum (DFS) [71] and Distance Learning Pegasos (DL-Pegasos) [22]. DFS can be seen as concatenation of two components: (i) a volumetric component that encodes the stochastic self-similarities of dynamic textures as 3D volumes, and (ii) a multi-slice dynamic component that captures structures of dynamic textures on $2 \mathrm{D}$ slices along various views of the 3D volume. DL-Pegasos uses three descriptors (LBP, HOG and LDS) and learns how the descriptors can be linearly combined to best discriminate between dynamic texture classes.

The overall classification results are presented in Table 7. The proposed $\mathrm{kgLC}$ with dictionary learning (kgLC-dic) obtains the highest average recognition rate.
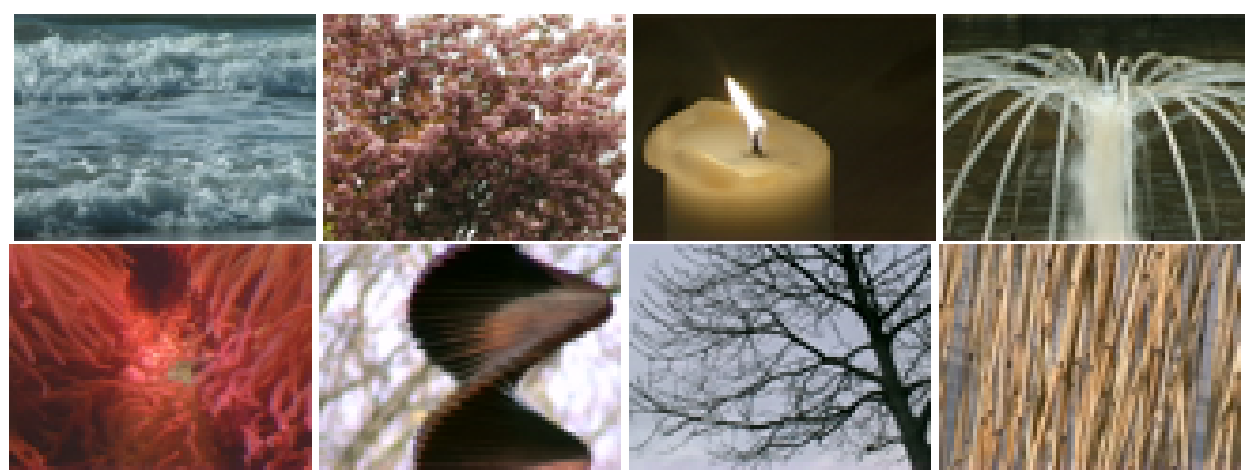

Figure 10: Example classes from the DynTex++ dataset (grayscale images were used in experiments).

Table 7: Average recognition rate on the DynTex++ dataset.

\begin{tabular}{lc}
\hline Method & CRR \\
\hline DL-PEGASOS [22] & 63.7 \\
DFS [71] & 89.9 \\
DCC [40] & 53.2 \\
KAHM [11] & 82.8 \\
GDA [30] & 81.2 \\
GGDA [33] & 84.1 \\
\hline gSC-dic & $\mathbf{9 0 . 3}$ \\
gLC-dic & $\mathbf{9 1 . 8}$ \\
kgSC-dic & $\mathbf{9 2 . 8}$ \\
kgLC-dic & $\mathbf{9 3 . 2}$ \\
\hline
\end{tabular}




\subsection{Computational Complexity}

Let $\mathbb{D}=\left\{\mathcal{D}_{i}\right\}_{i=1}^{N}, \mathcal{D}_{i} \in \mathcal{G}(p, d)$ be a Grassmannian dictionary and $\mathcal{X} \in \mathcal{G}(p, d)$ be a query sample with $\mathcal{D}_{j}=\operatorname{span}\left(\boldsymbol{D}_{j}\right)$ and $\mathcal{X}=\operatorname{span}(\boldsymbol{X})$. In terms of computational load, the gSC algorithm requires the values in the form of $\left\|\boldsymbol{X}^{T} \boldsymbol{D}_{j}\right\|_{F}^{2}$ which can be computed in $O\left(N d p^{2}\right)$ flops for the whole dictionary.

The iSC algorithm [36] solves (8) for coding. To this end, computing the logarithm map on $\mathcal{G}(p, d)$ is required. A very efficient implementation of the logarithm map on $\mathcal{G}(p, d)$ requires a matrix inversion of size $p \times p$, two matrix multiplications of size $d \times p$, and a thin SVD of size $d \times p$. Computing thin SVD using a stable algorithm like the Golub-Reinsch [24] requires $14 d p^{2}+8 p^{3}$ flops. This adds up to a total of $O\left(9 N p^{3}+16 N d p^{2}\right)$ flops for the whole dictionary.

To give the reader a better sense on the computational efficiency of gSC algorithm, we performed an experiment. Assuming that the complexity of vector sparse coding for both algorithms is similar (iSC is a constrained coding approach so it is very likely to be more expensive than an unconstrained one like gSC), we measured the time required to compute $\mathcal{K}_{\boldsymbol{X}}$ in Eq. (15) against projecting $\mathbb{D}$ to the tangent space of $\mathcal{X}$. To this end, we considered three cases using the geometry of $\mathcal{G}(3,100), \mathcal{G}(3,1000)$ and $\mathcal{G}(3,10000)$. We randomly generated a dictionary of size 1000 for each case and measured the time required to compute $\mathcal{K}_{\boldsymbol{X}}$ and tangent projection for 1000 query points. The results given in Table 8 show that the gSC algorithm is significantly faster than iSC.

Table 8: Running time comparison between the proposed gSC approach and intrinsic sparse coding (iSC) [36] method on synthetic data. Times are measured in second on a Quad-core i7 machine with Matlab.

\begin{tabular}{lccc}
\hline Task & $\mathcal{G}(3,100)$ & $\mathcal{G}(3,1000)$ & $\mathcal{G}(3,10000)$ \\
\hline iSC [36] & $77.8 \mathrm{~s}$ & $234.2 \mathrm{~s}$ & $1320.7 \mathrm{~s}$ \\
$\mathrm{gSC}$ & $4.1 \mathrm{~s}$ & $16.9 \mathrm{~s}$ & $106.4 \mathrm{~s}$ \\
\hline
\end{tabular}

\section{Main Findings and Future Directions}

With the aim of coding on Grassmann manifolds, we proposed to embed such manifolds into the space of symmetric matrices by an isometric projection. We then showed how sparse coding and locality linear coding can be performed in the induced space. We also tackled the problem of dictionary learning on Grassmann manifolds and devised a closed-form solution for updating a dictionary atom by atom, using the geometry of induced space. Finally, we proposed a kernelized version of sparse coding, locality linear coding and dictionary learning on Grassmann manifolds, to handle non-linearity in data.

Experiments on several classification tasks (gender recognition, gesture classification, scene analysis, face recognition, action recognition and dynamic texture classification) show that the proposed approaches achieve notable improvements in discrimination accuracy, in comparison to state-of-the-art methods such as discriminant analysis of canonical correlation analysis [40] affine hull method [11], Grassmann discriminant analysis [30], graph-embedding Grassmann discriminant analysis [33] and intrinsic sparse coding method [36].

In this work a Grassmann dictionary is learned such that a reconstruction error is minimized. This is not necessarily the optimum solution when labeled data is available. To benefit from labeled data, it has recently been proposed to consider a discriminative penalty term along with the reconstruction error term in the optimization process [45]. We are currently pursuing this line of research and seeking solutions for discriminative dictionary learning on Grassmann manifolds. Moreover, our formulation can be understood as an extrinsic solution to the problem of coding and dictionary learning on Grassmann manifolds. It would be interesting to devise intrinsic solutions based on the geometry of the induced space, i.e., symmetric matrices. 


\section{Acknowledgements}

NICTA is funded by the Australian Government as represented by the Department of Broadband, Communications and the Digital Economy, as well as the Australian Research Council through the ICT Centre of Excellence program. This work is funded in part through an ARC Discovery grant DP130104567. C. Shen's participation was in part supported by ARC Future Fellowship F120100969.

\section{A Appendix}

In this appendix, we give proofs for the following theorems.

Theorem A.1. Let $\boldsymbol{X}$ be an $d \times d$ symmetric matrix with eigenvalue decomposition $\boldsymbol{X}=\boldsymbol{U} \boldsymbol{D} \boldsymbol{U}^{T}$, where $\boldsymbol{D}$ contains the eigenvalues $\lambda_{i}$ of $\boldsymbol{X}$ in descending order. Let $\boldsymbol{U}_{p}$ be the $d \times p$ matrix consisting of the first $p$ columns of $\boldsymbol{U}$. Then $\widehat{\boldsymbol{U}}_{p}=\boldsymbol{U}_{p} \boldsymbol{U}_{p}^{T}$ is the closest matrix in $\mathcal{P} \mathcal{G}(p, d)$ to $\boldsymbol{X}$ (under the Frobenius norm).

Proof. Observe that $\|\widehat{\boldsymbol{V}}-\boldsymbol{X}\|_{F}^{2}=\|\widehat{\boldsymbol{V}}\|_{F}^{2}+\|\boldsymbol{X}\|_{F}^{2}-2\langle\widehat{\boldsymbol{V}}, \boldsymbol{X}\rangle$. Since $\|\widehat{\boldsymbol{V}}\|_{F}\left(\right.$ for $\widehat{\boldsymbol{V}} \in \mathcal{P} \mathcal{G}(p, d)$ ) and $\|\boldsymbol{X}\|_{F}$ are fixed, minimizing $\|\widehat{\boldsymbol{V}}-\boldsymbol{X}\|_{F}$ over $\widehat{\boldsymbol{V}} \in \mathcal{P} \mathcal{G}(p, d)$ is the same as maximizing $\langle\widehat{\boldsymbol{V}}, \boldsymbol{X}\rangle$. If $\widehat{\boldsymbol{V}}=\boldsymbol{V} \boldsymbol{V}^{T}$, we may write $\langle\widehat{\boldsymbol{V}}, \boldsymbol{X}\rangle=\operatorname{Tr}\left(\boldsymbol{V} \boldsymbol{V}^{T} \boldsymbol{X}\right)=\operatorname{Tr}\left(\boldsymbol{V}^{T} \boldsymbol{X} \boldsymbol{V}\right)$, so it is sufficient to maximize $\operatorname{Tr}\left(\boldsymbol{V}^{T} \boldsymbol{X} \boldsymbol{V}\right)$ over $\boldsymbol{V} \in \mathcal{G}(p, n)$.

If $\boldsymbol{X}=\boldsymbol{U} \operatorname{diag}\left(\lambda_{1}, \ldots, \lambda_{d}\right) \boldsymbol{U}^{T}$, then $\boldsymbol{U}_{p}^{T} \boldsymbol{X} \boldsymbol{U}_{p}=\operatorname{diag}\left(\lambda_{1}, \ldots, \lambda_{p}\right)$ and $\operatorname{Tr}\left(\boldsymbol{U}_{p}^{T} \boldsymbol{X} \boldsymbol{U}_{p}\right)=\sum_{i=1}^{p} \lambda_{i}$. On the other hand, let $\boldsymbol{W} \in \mathcal{G}(p, d)$. Then $\boldsymbol{W}^{T} \boldsymbol{X} \boldsymbol{W}$ is symmetric of dimension $p \times p$. Let $\mu_{1} \geq \mu_{2} \geq \ldots \geq \mu_{p}$ be its eigenvalues and $\boldsymbol{a}_{i}, i=1, \ldots, p$ the corresponding unit eigenvectors. Let $\boldsymbol{w}_{i}=\boldsymbol{W} \boldsymbol{a}_{i}$. Then the $\boldsymbol{w}_{i}$ are orthogonal unit vectors, and $\boldsymbol{w}_{i}^{T} \boldsymbol{X} \boldsymbol{w}_{i}=\mu_{i}$.

For $k=1$ to $p$, let $A_{k}$ be the subspace of $R^{d}$ spanned by $\boldsymbol{w}_{1}, \ldots, \boldsymbol{w}_{k}$ and $B_{k}$ be the space spanned by the eigenvectors $\boldsymbol{u}_{k}, \ldots, \boldsymbol{u}_{d}$ of $\boldsymbol{X}$. Counting dimensions, $A_{k}$ and $B_{k}$ must have non-trivial intersection. Let $\boldsymbol{v}$ be a non-zero vector in this intersection, and write $\boldsymbol{v}=\sum_{i=1}^{k} \alpha_{i} \boldsymbol{w}_{i}=\sum_{i=k}^{d} \beta_{i} \boldsymbol{u}_{i}$. Then

$$
\mu_{k} \leq \frac{\sum_{i=1}^{k} \alpha_{i}^{2} \mu_{i}}{\sum_{i=1}^{k} \alpha_{i}^{2}}=\frac{\boldsymbol{v}^{T} \boldsymbol{X} \boldsymbol{v}}{\boldsymbol{v}^{T} \boldsymbol{v}}=\frac{\sum_{i=k}^{d} \beta_{i}^{2} \lambda_{i}}{\sum_{i=k}^{d} \beta_{i}^{2}} \leq \lambda_{k} .
$$

Therefore $\mu_{k} \leq \lambda_{k}$ and $\operatorname{Tr}\left(\boldsymbol{W}^{T} \boldsymbol{X} \boldsymbol{W}\right)=\sum_{i=1}^{p} \mu_{i} \leq \sum_{i=1}^{p} \lambda_{i}=\operatorname{Tr}\left(\boldsymbol{U}^{T} \boldsymbol{X} \boldsymbol{U}\right)$.

The chordal mean. For two points (matrices) $\widehat{\boldsymbol{X}}$ and $\widehat{\boldsymbol{Y}}$ in $\mathcal{P} \mathcal{G}(p, d)$ the distance $\|\widehat{\boldsymbol{X}}-\widehat{\boldsymbol{Y}}\|_{F}$ is called the chordal distance between the two points. Given several points $\widehat{\boldsymbol{X}}_{i}$, the $\ell_{2}$ chordal mean of $\left\{\widehat{\boldsymbol{X}}_{i}\right\}_{i=1}^{m}$ is the element $\widehat{\boldsymbol{Y}} \in \mathcal{P} \mathcal{G}(p, d)$ that minimizes $\sum_{i=1}^{m}\left\|\widehat{\boldsymbol{Y}}-\widehat{\boldsymbol{X}}_{i}\right\|_{F}^{2}$. There is a closed-form solution for the chordal mean of a set of points in a Grassman manifold.

Theorem A.2. The chordal mean of a set of points $\widehat{\boldsymbol{X}}_{i} \in \mathcal{P G}(p, d)$ is equal to $\operatorname{Proj}\left(\sum_{i=1}^{m} \widehat{\boldsymbol{X}}_{i}\right)$.

Proof. The proof is analogous to the formula for the chordal mean of rotation matrices, given in [34]. By the same argument as in Theorem A.1, minimizing $\sum_{i=1}^{m}\left\|\widehat{\boldsymbol{X}}_{i}-\widehat{\boldsymbol{Y}}\right\|_{F}^{2}$ is equivalent to maximizing $\sum_{i=1}^{m}\left\langle\widehat{\boldsymbol{X}}_{i}, \widehat{\boldsymbol{Y}}\right\rangle=$ $\left\langle\sum_{i=1}^{m} \widehat{\boldsymbol{X}}_{i}, \widehat{\boldsymbol{Y}}\right\rangle$. Thus, the required $\widehat{\boldsymbol{Y}}$ is the closest point in $\mathcal{P} \mathcal{G}(p, d)$ to $\sum_{i=1}^{m} \widehat{\boldsymbol{X}}_{i}$, as stated. 


\section{References}

[1] P.-A. Absil, R. Mahony, and R. Sepulchre. Riemannian geometry of Grassmann manifolds with a view on algorithmic computation. Acta Applicandae Mathematica, 80(2):199-220, 2004.

[2] P.-A. Absil, R. Mahony, and R. Sepulchre. Optimization Algorithms on Matrix Manifolds. Princeton University Press, Princeton, NJ, USA, 2008.

[3] M. Aharon, M. Elad, and A. Bruckstein. K-svd: An algorithm for designing overcomplete dictionaries for sparse representation. IEEE Transactions on Signal Processing, 54(11):4311-4322, 2006.

[4] V. Arsigny, P. Fillard, X. Pennec, and N. Ayache. Log-Euclidean metrics for fast and simple calculus on diffusion tensors. Magnetic resonance in medicine, 56(2):411-421, 2006.

[5] R. Basri and D. W. Jacobs. Lambertian reflectance and linear subspaces. Pattern Analysis and Machine Intelligence, IEEE Transactions on, 25(2):218-233, 2003.

[6] E. Begelfor and M. Werman. Affine invariance revisited. In Proc. IEEE Conference on Computer Vision and Pattern Recognition (CVPR), pages 2087-2094, 2006.

[7] E. J. Candès, J. Romberg, and T. Tao. Robust uncertainty principles: exact signal reconstruction from highly incomplete frequency information. IEEE Transactions on Information Theory, 52(2):489-509, 2006.

[8] H. E. Cetingul and R. Vidal. Intrinsic mean shift for clustering on stiefel and grassmann manifolds. In Proc. IEEE Conference on Computer Vision and Pattern Recognition (CVPR), pages 1896-1902, 2009.

[9] H. E. Cetingul and R. Vidal. Sparse riemannian manifold clustering for HARDI segmentation. In IEEE International Symposium on Biomedical Imaging: From Nano to Macro, pages 1750-1753, 2011.

[10] H. E. Cetingul, M. J. Wright, P. M. Thompson, and R. Vidal. Segmentation of high angular resolution diffusion MRI using sparse Riemannian manifold clustering. IEEE Transactions on Medical Imaging, 33(2):301-317, Feb 2014.

[11] H. Cevikalp and B. Triggs. Face recognition based on image sets. In Proc. IEEE Conference on Computer Vision and Pattern Recognition (CVPR), pages 2567-2573, 2010.

[12] A. B. Chan and N. Vasconcelos. Probabilistic kernels for the classification of auto-regressive visual processes. In Proc. IEEE Conference on Computer Vision and Pattern Recognition (CVPR), pages 846-851, 2005.

[13] S. Chen, C. Sanderson, M. Harandi, and B. C. Lovell. Improved image set classification via joint sparse approximated nearest subspaces. In Proc. IEEE Conference on Computer Vision and Pattern Recognition (CVPR), pages 452-459, 2013.

[14] Y. Chikuse. Statistics on Special Manifolds, volume 174. Springer, 2003.

[15] K. D. Cock and B. D. Moor. Subspace angles between ARMA models. Systems and Control Letters, 46:265-270, 2002.

[16] N. Dalal and B. Triggs. Histograms of oriented gradients for human detection. In Proc. IEEE Conference on Computer Vision and Pattern Recognition (CVPR), pages 886-893, 2005.

[17] D. L. Donoho. Compressed sensing. IEEE Transactions on Information Theory, 52(4):1289-1306, 2006.

[18] G. Doretto, A. Chiuso, Y. N. Wu, and S. Soatto. Dynamic textures. Int. Journal of Computer Vision, 51:91-109, 2003.

[19] M. Elad. Sparse and Redundant Representations - From Theory to Applications in Signal and Image Processing. Springer, 2010.

[20] E. Elhamifar and R. Vidal. Sparse subspace clustering: Algorithm, theory, and applications. IEEE Transactions on Pattern Analysis and Machine Intelligence, 35(11):2765-2781, 2013.

[21] K. A. Gallivan, A. Srivastava, X. Liu, and P. Van Dooren. Efficient algorithms for inferences on Grassmann manifolds. In IEEE Workshop on Statistical Signal Processing, pages 315-318, 2003.

[22] B. Ghanem and N. Ahuja. Maximum margin distance learning for dynamic texture recognition. In Proc. European Conference on Computer Vision (ECCV), volume 6312, pages 223-236, 2010.

[23] A. Goh and R. Vidal. Clustering and dimensionality reduction on Riemannian manifolds. In Proc. IEEE Conference on Computer Vision and Pattern Recognition (CVPR), pages 1-7, 2008.

[24] G. H. Golub and C. F. Van Loan. Matrix computations (3rd ed.). Johns Hopkins University Press, Baltimore, MD, USA, 1996.

[25] B. Gong, Y. Shi, F. Sha, and K. Grauman. Geodesic flow kernel for unsupervised domain adaptation. In Proc. IEEE Conference on Computer Vision and Pattern Recognition (CVPR), pages 2066-2073, 2012.

[26] R. Gopalan, R. Li, and R. Chellappa. Unsupervised adaptation across domain shifts by generating intermediate data representations. IEEE Transactions on Pattern Analysis and Machine Intelligence, 36(11):2288-2302, 2014.

[27] M. Grant and S. Boyd. Graph implementations for nonsmooth convex programs. In Recent Advances in Learning and Control, Lecture Notes in Control and Information Sciences, pages 95-110. Springer-Verlag Limited, 2008.

[28] M. Grant and S. Boyd. CVX: Matlab software for disciplined convex programming, version 2.0 beta. http: / / cvxr. com/cvx, Sept. 2012. 
[29] K. Guo, P. Ishwar, and J. Konrad. Action recognition from video using feature covariance matrices. IEEE Transactions on Image Processing (TIP), 22(6):2479-2494, 2013.

[30] J. Hamm and D. D. Lee. Grassmann discriminant analysis: a unifying view on subspace-based learning. In Proc. Int. Conference on Machine Learning (ICML), pages 376-383, 2008.

[31] M. Harandi, C. Sanderson, C. Shen, and B. C. Lovell. Dictionary learning and sparse coding on Grassmann manifolds: An extrinsic solution. In Proc. Int. Conference on Computer Vision (ICCV), December 2013.

[32] M. T. Harandi, R. Hartley, B. C. Lovell, and C. Sanderson. Sparse coding on symmetric positive definite manifolds using Bregman divergences. IEEE Transactions on Neural Networks and Learning Systems (TNNLS), (in press). http://dx.doi.org/10.1109/TNNLS.2014.2387383

[33] M. T. Harandi, C. Sanderson, S. Shirazi, and B. C. Lovell. Graph embedding discriminant analysis on Grassmannian manifolds for improved image set matching. In Proc. IEEE Conference on Computer Vision and Pattern Recognition (CVPR), pages 2705-2712, 2011.

[34] R. Hartley, J. Trumpf, Y. Dai, and H. Li. Rotation averaging. Int. Journal of Computer Vision, 103(3):267-305, 2013.

[35] U. Helmke, K. Hüper, and J. Trumpf. Newtons method on Grassmann manifolds. Preprint: arXiv:0709.2205, 2007.

[36] J. Ho, Y. Xie, and B. Vemuri. On a nonlinear generalization of sparse coding and dictionary learning. In Proc. Int. Conference on Machine Learning (ICML), pages 1480-1488, 2013.

[37] H. Karcher. Riemannian center of mass and mollifier smoothing. Communications on Pure and Applied Mathematics, 30(5):509-541, 1977.

[38] M. Kim, S. Kumar, V. Pavlovic, and H. Rowley. Face tracking and recognition with visual constraints in real-world videos. In Proc. IEEE Conference on Computer Vision and Pattern Recognition (CVPR), pages 1-8, 2008.

[39] T.-K. Kim and R. Cipolla. Canonical correlation analysis of video volume tensors for action categorization and detection. IEEE Transactions on Pattern Analysis and Machine Intelligence, 31(8):1415-1428, 2009.

[40] T.-K. Kim, J. Kittler, and R. Cipolla. Discriminative learning and recognition of image set classes using canonical correlations. IEEE Transactions on Pattern Analysis and Machine Intelligence, 29(6):1005-1018, 2007.

[41] E. Kokiopoulou, J. Chen, and Y. Saad. Trace optimization and eigenproblems in dimension reduction methods. Numerical Linear Algebra with Applications, 18(3):565-602, 2011.

[42] J. M. Lee. Introduction to smooth manifolds, volume 218. Springer, 2012.

[43] B. Li, M. Ayazoglu, T. Mao, O. I. Camps, and M. Sznaier. Activity recognition using dynamic subspace angles. In Proc. IEEE Conference on Computer Vision and Pattern Recognition (CVPR), pages 3193-3200, 2011.

[44] Y. M. Lui. Human gesture recognition on product manifolds. Journal of Machine Learning Research, 13:3297-3321, 2012.

[45] J. Mairal, F. Bach, and J. Ponce. Task-driven dictionary learning. IEEE Transactions on Pattern Analysis and Machine Intelligence, 34(4):791-804, 2012.

[46] J. Mairal, F. Bach, J. Ponce, and G. Sapiro. Online learning for matrix factorization and sparse coding. Journal of Machine Learning Research, 11:19-60, 2010.

[47] J. Mairal, F. Bach, J. Ponce, G. Sapiro, and A. Zisserman. Discriminative learned dictionaries for local image analysis. In Proc. IEEE Conference on Computer Vision and Pattern Recognition (CVPR), pages 1-8. IEEE, 2008.

[48] J. Mairal, M. Elad, and G. Sapiro. Sparse representation for color image restoration. IEEE Transactions on Image Processing (TIP), 17(1):53-69, 2008.

[49] J. H. Manton. A globally convergent numerical algorithm for computing the centre of mass on compact lie groups. In Int. Conf. on Control, Automation, Robotics and Vision, volume 3, pages 2211-2216, 2004.

[50] T. Ojala, M. Pietikäinen, and T. Mäenpää. Multiresolution gray-scale and rotation invariant texture classification with local binary patterns. IEEE Transactions on Pattern Analysis and Machine Intelligence, 24:971-987, July 2002.

[51] B. A. Olshausen and D. J. Field. Emergence of simple-cell receptive field properties by learning a sparse code for natural images. Nature, 381(6583):607-609, 1996.

[52] R. Ramamoorthi. Analytic PCA construction for theoretical analysis of lighting variability in images of a Lambertian object. IEEE Transactions on Pattern Analysis and Machine Intelligence, 24(10):1322-1333, Oct 2002.

[53] S. R. Rao, R. Tron, R. Vidal, and Y. Ma. Motion segmentation via robust subspace separation in the presence of outlying, incomplete, or corrupted trajectories. In Proc. IEEE Conference on Computer Vision and Pattern Recognition (CVPR), pages 1-8. IEEE, 2008.

[54] A. Ravichandran, P. Favaro, and R. Vidal. A unified approach to segmentation and categorization of dynamic textures. In Proc. Asian Conference on Computer Vision (ACCV), pages 425-438. Springer, 2011.

[55] S. T. Roweis and L. K. Saul. Nonlinear dimensionality reduction by locally linear embedding. Science, 290(5500):2323-2326, 2000. 
[56] C. Sanderson, M. T. Harandi, Y. Wong, and B. C. Lovell. Combined learning of salient local descriptors and distance metrics for image set face verification. In Proc. Int. Conf. Advanced Video and Signal-Based Surveillance, pages 294-299, 2012.

[57] A. Sankaranarayanan, P. Turaga, R. Baraniuk, and R. Chellappa. Compressive acquisition of dynamic scenes. In Proc. European Conference on Computer Vision (ECCV), volume 6311, pages 129-142, 2010.

[58] J. Shawe-Taylor and N. Cristianini. Kernel Methods for Pattern Analysis. Cambridge University Press, 2004.

[59] S. Shirazi, C. Sanderson, C. McCool, and M. T. Harandi. Bags of affine subspaces for robust object tracking. Preprint: arXiv:1408.2313, 2015.

[60] A. Srivastava and E. Klassen. Bayesian and geometric subspace tracking. Advances in Applied Probability, 36(1):43-56, 2004.

[61] R. Subbarao and P. Meer. Nonlinear mean shift over Riemannian manifolds. Int. Journal of Computer Vision, 84(1):1-20, 2009.

[62] R. Tibshirani. Regression shrinkage and selection via the lasso. Journal of the Royal Statistical Society. Series $B$ (Methodological), pages 267-288, 1996.

[63] P. Turaga, A. Veeraraghavan, A. Srivastava, and R. Chellappa. Statistical computations on Grassmann and Stiefel manifolds for image and video-based recognition. IEEE Transactions on Pattern Analysis and Machine Intelligence, 33(11):2273-2286, 2011.

[64] M. Turk and A. Pentland. Eigenfaces for recognition. Journal of Cognitive Neuroscience, 3(1):71-86, 1991.

[65] R. Vemulapalli, J. K. Pillai, and R. Chellappa. Kernel learning for extrinsic classification of manifold features. In Proc. IEEE Conference on Computer Vision and Pattern Recognition (CVPR), pages 1782-1789, 2013.

[66] P. Viola and M. J. Jones. Robust real-time face detection. Int. Journal of Computer Vision, 57(2):137-154, 2004.

[67] J. Wang, J. Yang, K. Yu, F. Lv, T. Huang, and Y. Gong. Locality-constrained linear coding for image classification. In Proc. IEEE Conference on Computer Vision and Pattern Recognition (CVPR), pages 3360-3367, 2010.

[68] Y. Wang and G. Mori. Human action recognition by semilatent topic models. IEEE Transactions on Pattern Analysis and Machine Intelligence, 31(10):1762-1774, 2009.

[69] J. Wright, Y. Ma, J. Mairal, G. Sapiro, T. S. Huang, and S. Yan. Sparse representation for computer vision and pattern recognition. Proceedings of the IEEE, 98(6):1031-1044, 2010.

[70] J. Wright, A. Y. Yang, A. Ganesh, S. S. Sastry, and Y. Ma. Robust face recognition via sparse representation. IEEE Transactions on Pattern Analysis and Machine Intelligence, 31(2):210-227, 2009.

[71] Y. Xu, Y. Quan, H. Ling, and H. Ji. Dynamic texture classification using dynamic fractal analysis. In Proc. Int. Conference on Computer Vision (ICCV), 2011.

[72] J. Yang, K. Yu, Y. Gong, and T. Huang. Linear spatial pyramid matching using sparse coding for image classification. In Proc. IEEE Conference on Computer Vision and Pattern Recognition (CVPR), pages 1794-1801, 2009.

[73] K. Yu and T. Zhang. Improved local coordinate coding using local tangents. In Proc. Int. Conference on Machine Learning (ICML), pages 1215-1222, 2010.

[74] K. Yu, T. Zhang, and Y. Gong. Nonlinear learning using local coordinate coding. In Proc. Advances in Neural Information Processing Systems (NIPS), volume 9, page 1, 2009.

[75] S. Yu, T. Tan, K. Huang, K. Jia, and X. Wu. A study on gait-based gender classification. IEEE Transactions on Image Processing (TIP), 18(8):1905-1910, 2009.

[76] C. Yuan, W. Hu, X. Li, S. Maybank, and G. Luo. Human action recognition under log-Euclidean Riemannian metric. In H. Zha, R.-i. Taniguchi, and S. Maybank, editors, Proc. Asian Conference on Computer Vision (ACCV), volume 5994 of Lecture Notes in Computer Science, pages 343-353. Springer Berlin Heidelberg, 2010.

[77] G. Zhao and M. Pietikäinen. Dynamic texture recognition using local binary patterns with an application to facial expressions. IEEE Transactions on Pattern Analysis and Machine Intelligence, 29(6):915-928, 2007.

[78] S. Zheng, J. Zhang, K. Huang, R. He, and T. Tan. Robust view transformation model for gait recognition. In International Conference on Image Processing (ICIP), pages 2073-2076, 2011. 